\title{
Blockchain technology applications for power systems: current trends and future applications
}

\author{
Maria Luisa Di Silvestre', Pierluigi Gallo', Josep M. Guerrero' ${ }^{2}$, Rossano Musca ${ }^{1}$, \\ Eleonora Riva Sanseverinoㄹ, Giuseppe Sciumè', Juan C. Vásquez², Gaetano Zizzo' \\ 1. University of Palermo, Palermo (Italy) \\ 2. Aalborg University, Aalborg (Denmark)
}

\begin{abstract}
Today, the blockchain is synonymous of technological innovation, being recognised among the 10 top strategy technologies in 2018 by the consulting company Gartner, it is more and more adopted in different sectors. However, the initial enthusiasm around this technology is going beyond the peak of inflated expectations, towards more stable applications in money transactions, cryptocurrencies and Digital Commodity Exchanges. Essentially, misguided efforts, the overuse of blockchain, and the Bitcoin's price drop have been the main reasons for this decay in expectations. Nevertheless, the exploitation of the blockchain technology in the power systems area appears largely underexplored, furthermore, the relation to the physical asset makes the blockchain application more complex but also more reliable and related to measurable benefits. The most common applications in the power systems area relate to the energy market. When the blockchain technology is indeed applied to the energy field, the term energy blockchain is used. This article aims to propose a wide perspective about the application of the blockchain technology in the power systems area, clarifying some technical aspects concerning this promising technology, the features and applications developed so far, while focusing on the future of innovative applications in the electrical energy sector.
\end{abstract}

Keywords: Peer-to-peer; Energy blockchain; Energy Market; Supply chain management; Aggregation; Decentralized Control; Decentralized Management; Review.

\section{Introduction}

Since 2008, when the first and most famous blockchain application (for transactions in BitCoin) was created, many companies have tried to use this technology for applications in various sectors, such as: finance, electronic voting (e-voting), copyright and patent management, smart contracts, tracking of goods (supply chain) and energy market. Blockchain is, indeed, not the synonymous of Bitcoin. The latter is the most famous crypto-currency in the world. Bitcoin was founded in 2008 with the aim of creating an electronic payment system that allows two counterparties to negotiate directly with each other without the need for a third party of trust. The traditional Internet commerce system relies on financial institutions, which serve as a means of processing electronic payments. This system suffers from the inherent weaknesses of a model based on the trust of unknown users towards a third party that acts as the guarantor for both. This results in a brokerage cost that increases the transaction cost, limiting the minimum size of transactions that can be carried out and excluding the possibility of small transactions. Bitcoin was created to cope with these problems, proposing a new technology based on cryptographic evidence instead of trust, which allows the exchange of currency between two unknown users without the need for a third party to act as guarantor for both. The new cutting-edge technology used by the Bitcoin application is precisely the blockchain. The latter allows to record events ordered in time and ensure that the recorded events are difficult to delete or modify even if some malicious users try to do it, since every change requires many resources and/or the approval of most of the network nodes and not of 
a single authority as in the case of the classic centralized systems. Therefore, users have not to rely on third parties, which often make decisions for their own interests. For this reason, it is particularly useful in cases where two people want to make a deal, but do not trust each other [1]

Before the advent of blockchain, the transactions of any good were recorded in a centralized database managed by an institution, which can do any operation on the data, and therefore has to be trusted to avoid misbehavior on data. In the financial field, this central role is run by the bank, in the electrical energy world the central role is played by different entities depending on the type of transaction. A third-party operator manages all financial and energy transactions in the wholesale energy market (GME in Italy, CRE in France, etc.), while the Transmission System Operator (TSO) manages all the financial and energy transactions in the balancing market (Terna in Italy, RTE in France, etc.) checking the technical feasibility of each one. Moreover, recent indications from ENTSO-E, are stressing the more and more important role of Distribution System Operators (DSO) for managing the flexibility of local resources connected to their grids in a more extended flexibility market [76]. These transactions (financial or electric energy transactions) are in any case recorded in a database. (R2-1.1) The novelty introduced by the blockchain is the distribution of data and trust in the network while overcoming the problems afflicting decentralized systems. Table 1 shows an overview of the main advantages and disadvantages of centralized and distributed database systems [2], [3]. (R2-1.2)

In distributed systems, each node has the same capabilities and the same level of access to complete information. This provides a solid foundation for trust, as it democratizes the entire system. Furthermore, since the data is stored on all the nodes of the system, in the event of a cyber-attack there is no single point of failure, so the system can continue working. On the other hand, in a traditional centralized system (database), one relies on a single central authority that controls "who can do what" in the system. This latter type of system is more vulnerable in the event of a cyber-attack, but it is good when the party that controls the system is reliable and behaves honestly. In general, distributed system, and so the blockchain, is more suitable in cases where there is more than one administrative authority and there is a lack of trust between these parties (R2-1.2) [3].

Table 1. Advantages and disadvantages of centralized and distributed systems.

\begin{tabular}{|c|c|c|}
\hline System & Advantages & Disadvantages \\
\hline Centralized & $\begin{array}{l}\text { - Very efficient system; } \\
\text { - The decision-making process is quick } \\
\text { and effective; } \\
\text { - There are no duplications; } \\
\text { - The bureaucratic apparatus is more } \\
\text { efficient. }\end{array}$ & $\begin{array}{l}\text { - Power and authority are concentrated in } \\
\text { the hands of the central government; } \\
\text { - There is not a public and democratic } \\
\text { participation; } \\
\text { - Applications run on a single process that } \\
\text { is the only component of the system; } \\
\text { - The component constitutes the single } \\
\text { point of failure of the system. }\end{array}$ \\
\hline
\end{tabular}




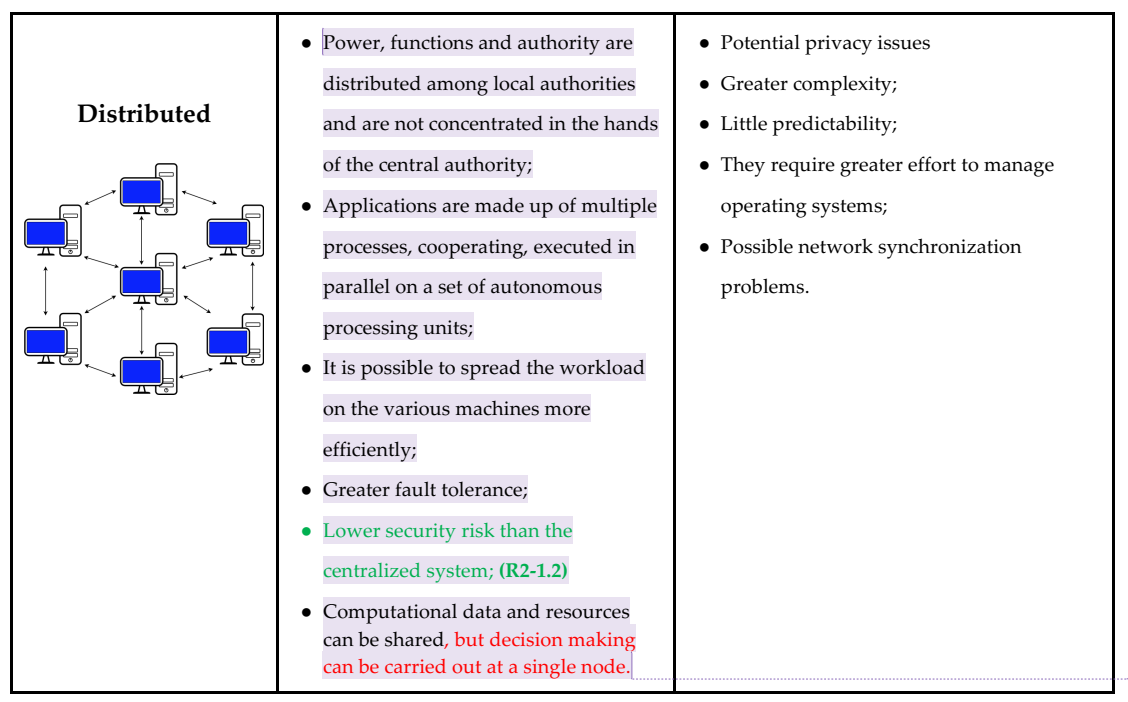

Another unquestionable benefit of the blockchain technology is the inherent integration of communication protocol in Business to Business [4] (B2B) and Business to Consumer (B2C) applications. In a multi-party environment with $\mathrm{N}$ actors, such as electrical energy applications are (i.e. trading is one example), there is a high number of connections. What if such connections are designed with the same communication technology? That would reduce the integration costs a lot both in B2B and B2C environments. In B2B applications for electricity trading in power systems, such as the day-ahead energy market, the communication technologies currently used are many. Such as many are the platforms for trading. As an example, the EPEX SPOT is a platform that matches orders in a transparent manner to all parties, however, it is only one of the involved communication technologies, since many others are used to transfer this information to the European Commodity Clearing (ECC) and for the latter to take obligations from buyer and seller. Communication to the TSO from ECC will have to be made too, and this may take place through further communication technology [5]. By adopting blockchain technology, the three main dimensions of B2B integration (data format, business process and communication protocol) would thus keep balanced, giving the dimension of the communication protocol the right importance making it homogeneous for all parties.

On the other hand, in B2C, transparency over supply chains would provide consumers a view over the products or services purchased.

In general, a blockchain can thus be defined as a distributed transaction ledger, structured in blocks and managed by network nodes. The blockchain can be public or private depending on the access to the ledger, whereas it is named permissioned or permissionless depending on who is authorized to maintain the ledger [51]. In a nutshell, a blockchain is a chain of blocks, each block contains several transactions, and the whole information is shared among all the nodes participating in the blockchain. Each node can see all transactions, thus creating a network that allows the traceability of all transactions. In this way, whatever is their role (consumers, companies, certification authorities, operators, ...) network participants are fully accountable for their actions and contracts with each other [6]. Each block is connected to the previous one and to the next one through the use of cryptographic hash functions. The hash functions are particular functions that allow converting a
Commentato [ER1]: @giuseppe @pierluigi Secondo voi è coerente quanto scritto con quanto riportato qui?
https://medium.com/distributed-economy/what-is-the$\mathrm{https}: /$ medium.com/distributed-economy/what-is-the-
difference-between-decentralized-and-distributed-systemsf4190a5c6462

Commentato [ER2]: Ho invertito I paragrafi. Mi sembrava più logico 
message of any length, in this case the data related to a block, to a message of fixed length (a digest named hash) that identifies it univocally. Furthermore, it is not easy to find two messages that have the same hash, this property is named collision resistance. In other words, they create a string associated with the data of the reference block for which, once the function is applied, it is no longer possible to return to the starting data. The peculiarity of these functions is that they are easy to calculate and always give the same result using the same input data. Blockchain technology exploits these functions to create the chain of blocks: on each block, there is a hash calculated using the transaction data contained in the block itself plus the hash of the previous block. In this way, each block stores information (the hash of the previous block) from all the transactions that are on all the nodes of the network and, thanks to the chaining system, it is unmodifiable (if not, through the repetition of all the network blocks). The blockchain goes beyond the functions of a distributed database, i.e. a database that is not physically located on a single server (computer), but instead is located on multiple computers at the same time, all perfectly synchronized on the same documents. In fact, unlike a database, blockchain transactions are validated before becoming part of new blocks, requiring approval from most of the nodes participating in the network, and, once confirmed, it becomes difficult to alter as it in turn requires an incredibly high computational power or the approval of most network nodes [7]. Figure 1 shows the whole process to insert a new transaction in the blockchain. The process is divided in six steps:

1. A request of transaction;

2. The new request of the transaction is sent to the network;

3. The transaction is sent to the nodes responsible for transactions' validation;

4. If the transaction is valid, the validator node inserts the new transaction in a new block containing other valid transactions and compete to validate the block (consensus process);

5. After the validation of the new block, the successful validator can insert the new block on the chain;

6. Once the new block is part of the chain, all participants will be able to see its content.

Furthermore, thanks to decentralization, it is possible to prevent market abuse through monopolies and reduce costs and regulatory oversight.

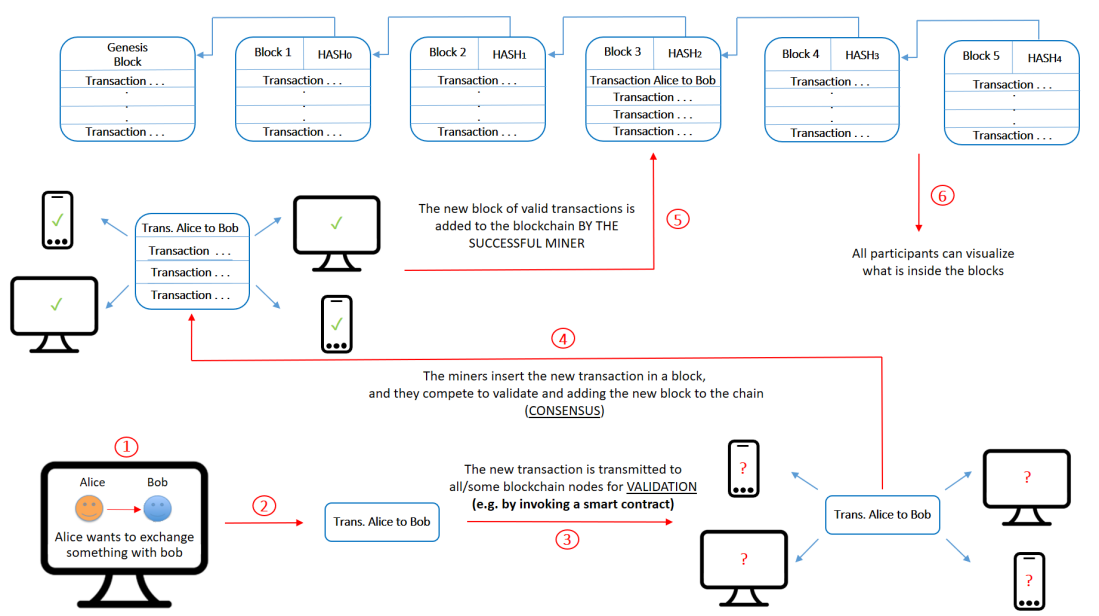

Figure 1. How a blockchain with validation and consensus works. 
The nodes that provide the validation of transactions and blocks are called 'miners'. The validation of the transaction can be carried out using particular cryptographic functions or by running a 'smart contract', instead, the validation of a new block by using specific algorithms called 'consensus algorithms'. Details on smart contracts, the different consensus algorithms and the process for achieving consensus over a block of transactions are reported, with specific reference, in later sections. This feature provides the authoritative validation that in a centralized system is guaranteed by the trusted authority or more in general by the system manager.

The use of the blockchain technology thus allows to obtain:

1. Transparency (in public blockchain), because each block added to the blockchain is accessible to all participants and is in the archive of all participants.

2. Immutability, since a block, thanks to the use of hash functions, once added, can be changed only having an extremely high computing power in case of permissionless blockchain (more than the one used for mining the single block) or with the approval of most of the participants in the network in the case of permissioned blockchain.

3. Confidence, since there is a shared reading among all the participants;

4. Efficiency (to be evaluated case by case), since it can not require intermediaries than the classic transaction management system, thus simplifying processes, infrastructures and increasing operational efficiency;

5. Control and security, since it allows the use of cryptography allowing greater data protection and lower risk of fraud.

As already mentioned, the blockchain is not the only alternative for storing a small amount of data of transactions and using them. The work in [8] offers a flow diagram that allows assessing whether the considered application really needs a blockchain-based approach or not. In the flow diagram, a few features are considered 'basic requirements' for applying blockchain. These are:

- multiparty;

- decentralized operation;

- need for transactions history:

- no need for high speed for the process.

Another important technology the blockchain allows to implement is the so-called 'smart contract'. Smart contracts are codes shared on the blockchain network that are executed to implement the logic of the transactions and verify their validity. They are also used to execute transactions automatically if certain conditions are met [9]. They thus allow the property transfer of any digitally identifiable good from one person to another. In peer-to-peer (P2P) electricity transactions as an example, the smart contract allows the transfer of tokens from one party to another and 'certifies' the activation of a local resource at the premises of one of the two parties while acquiring registrations of the consumptions and injections.

Many recent works deal with P2P energy transactions in power systems and with the energy blockchain. As an example, the works in [10], [11] offers a state of the art for P2P energy transactions, but they do not focus on blockchain and on its peculiar features. Same for [12] and [13], which focus on P2P trading considering federated power plants and on a wide state of the art about P2P trading models and technologies. The work in [14] is still a review of the existing P2P trading projects, which analyze the compatibility of the Energy Internet and blockchain technology but considering only the technical aspects of the blockchain for P2P trading. In [15] and [16] the 
authors give a broader overview of the suitability of blockchain technology applied to energy systems, taking into account the blockchain features, advantages and disadvantages, the ongoing projects, but focusing only on energy trading applications. Regarding the potential application of the blockchain technology in modern power systems, the work in [17] shows the various advantages that could be obtained in terms of flexibility and operational solutions, while the work in [18] shows the advantages that could be obtained in terms of cybersecurity. Finally, the work in [19] reports the summary of some interviews of experts on the future applications of the energy blockchain while in [20] the implications for policy developments are discussed.

Research methodology: The present paper starts from an in-depth analysis of the scientific articles and technical reports dealing with the current and feasible applications of the blockchain technology to power systems at all levels (generation, transmission, distribution, market, final usage, etc.). The research methodology applied in this study was devoted to the examination of these documents available in the literature for finding what aspects are still not discussed on this subject. (R2-1.4)

Contribution of the paper: Therefore, in this paper, starting from the critical examination of the above works, a comprehensive state of the art of blockchain-based applications for power systems is offered. Differently from other works, the current paper is not only devoted to examine P2P energy transactions. In particular, this paper discusses the pros and cons of the use of blockchain technology in the various power systems applications considering their peculiar features. The novelty aspects of this work are fundamentally two:

- for each class of applications, the paper identifies the most suitable technology, according to the analysis of the different available blockchain technologies and smart contract implementations;

- the paper tries to solve a very practical question: the identification of those situations where blockchain can be really useful for power systems applications and where, instead, its implementation can be avoided. (R2-1.4)

Structure of the paper: The document is organized as follows. Section 2 describes the blockchain applications to power systems. Section 3 contains a discussion about the features and technical requirements of the blockchain technology for various applications in the power systems area. Section 4 reports the current experiences of blockchain applications to power systems. Section 5 (R2-1.3) contains a discussion of the future developments and trends, with a focus on the new European electricity market and on the open challenges. Finally, section 6 presents the conclusions of the paper.

\section{Blockchain applications in power systems}

In the energy field, there are many possible applications of the blockchain technology, and most of these are aiming at P2P energy trading [10], [21], [22], [23]. In the field of power systems, the parties, however, may change, these being Electric Vehicles [24], [25], Manageable loads [26], Producers or Prosumers, Energy Storage Systems [27].

There are other popular applications of the blockchain technology to the power sector. As far as electrical power applications are concerned, they can indeed be divided into two main classes:

- Electrical energy Trading;

- Renewable Energy Certification and Demand Response tracing.

The first class comprises all applications for which two parties exchange electricity against a unit of value, including P2P energy trading applications. These can be either managed centrally by a grid operator (DSO or TSO) that will match exchanges in order to get the maximum benefit in technical terms for the grid or can be managed in a distributed fashion (grid constraints are not so tight).

The second class comprises Renewable Energy Certificates emissions applications and Aggregation/Demand Response tracing. In this case, the blockchain records the injection of green 
power at one site into the grid or recognizes the participation of a given prosumer in an aggregation program or a demand response program, improving transparency on renewable energy certificates emission or remuneration mechanisms. In what follows the two classes are detailed further and a short review is provided.

\subsection{Electrical energy trading}

The Energy Blockchain has become a very famous technology for enhancing P2P electrical energy trading between producers and consumers in microgrids. Some recent papers focus on this application [28], [29], [30], [31] proposing a generic blockchain-based framework to facilitate peerto-peer electricity sales in microgrids. Such as in all cyber-physical application, verification of the validity of the transaction also relies on the reliability of the smart counter and all apparatus that certify measurements and operations. Some P2P platforms aim at providing energy balance downstream substations [32], [33]. However, although energy balance is provided downstream a transformer, reactive power flows may appear as a result of voltage-regulated buses (i.e.: Voltage Source Converters interfacing PV, wind or storage units) [34]. Many papers [35], [36] do not consider these issues, thus on the one hand, not exploiting the potential of reducing balancing and network costs overhead on consumers, on the other, not accounting for the certification of smart meters readings which are some of the physical assets that must be considered in the cyberphysical implementation of the blockchain. Finally, it can be observed through the analysis of the applications that if technical feasibility of transactions must be verified, the grid operator takes a 'certifier' role that cannot be surrogated by any 'shared' verification mechanisms. In the section dedicated to the applications in power systems different examples about the two implementations are provided.

The paper in [37] proposes a P2P electricity trading algorithm that selects the best matches between demands and offers along a 24 hours timeframe to minimize unbalances at the MV/LV transformer. However, unless the algorithm is part of a smart contract registered over a Blockchain, the proposed algorithm could be part of the strategy of an aggregator rather than a purely P2P technique. Moreover, it seems unrealistic that all nodes have access to the readings of all meters and thus the role of the DSO comes into play also for this application. A more recent paper from the same authors [38], indeed, proposes an architecture for electricity trading that resembles realistic technical problems and includes the DSO having a main role. In this way, the balancing problems can be faced and efficiently managed.

The paper in [39] proposes an authorized blockchain that uses hyperledger fabric to provide a network of P2P energy transactions in order to cope with the growing volume of renewable energy, using Energy Storage System (ESS), considering the benefits obtainable from consumers but without taking into account the repercussions that can be had on the network. The work in [40] proposes an energy trading platform using also ESS and energy token exchange but to solve the security problem through the blockchain private network and implement the P2P system that enhances the transparency and immutability by executing the automatic transaction without involving the third party using the smart contract. The proposed architecture consists of a private blockchain network in which energy is exchanged through ESS and energy tokens through the blockchain. Users register sales and purchase offers on the blockchain, after which the DSO compares the total amount of the purchase and sale. Trade is realized if the amount of energy to buy and sell is equal. So the smart contract moves the token and the energy according to the accomplished trade the result is recorded on blockchain.

With the aim of improving demand management, P2P energy trading processes and security, but still without taking into account the technical aspects of the network, the paper in [41] proposes a double-chain model, which allows avoiding sophistication in data management. 


\subsection{Renewable Energy Certification and Demand Response tracing}

Apart from the application to energy transactions, blockchain promises to be an attractive solution for some issues regarding the transition of the classical electrical power system towards a decentralized structure. The advent of the blockchain in the energy sector integrates well with trend in decentralized generation through the widespread implementation of photovoltaic plants, other distributed generators and ICT devices in power systems. The blockchain technology offers new opportunities related to the certification of the production from RES, its tracing, the control of the grid, the coordination of the distributed resources (generators, batteries, flexible loads, etc.), the protection of the system (against overcurrents, reverse power flow phenomena, excessive voltage rising, detachment of generators and lacks of production, etc.) and the participation of the prosumers in regulation and electricity markets. Many of the problems that grid operators (DSO and TSO) are currently facing are related specifically to:

- participation of small prosumers in Demand Response (DR) programs;

- aggregation of local resources for participating in the Capacity and Balancing Markets;

- need for new ancillary services from distributed units;

- supervision and coordination of the aforementioned processes.

The exchange of information and control signals has become a fundamental part of the decentralized power system, able to maintain or threaten the security of the grid. In addition, the huge number of distributed resources has made almost impossible the implementation of direct control of the devices and generators operated by the utility. In this way, owners are called to the management of their own energy resources [42] in Demand Response schemes.

In this context, the blockchain technologies offer new ways of contributing to the development of future power systems. In the most recent scientific literature and in the current applications done by aggregators, looking at the same problems of aggregation and DR implementation from the grid operator perspective, applications of the blockchain can be found in the following areas:

- P2P decentralized management of Demand Response in the grid;

- Demand Response applications and distributed resources aggregation;

- Incentive mechanisms for prosumers in Demand Response schemes.

The three issues are often correlated in the smart grid paradigm. The issue of the decentralized management of DR in microgrids is discussed in many recent papers aiming at proposing new control models of microgrids, not comprising a central controller exchanging data with all distributed resources. As an example, in [27] the blockchain is used for storing in a tamper-proof manner the energy usage and generation information collected from the prosumers' smart meters, and smart contracts evaluate the expected flexibility of each prosumer, the associated remuneration or penalty, and the rules for the whole microgrid's energy balancing. The literature offers a wide variety of papers that although not explicitly referring to blockchain technology offer quite suitable applications for this technology. All P2P applications in a trustless environment indeed require transparency and security. In [43], a fully decentralized control system, using the ICT concept of network overlays and P2P networks for microgrids is proposed. The work shows how decentralization can be achieved using P2P frameworks as underlying control structures. In [47], a solution based on a Virtual Aggregation Environment for providing flexibility to the DSO without 
an intermediate Balancing Service Provider is proposed. In this case, the DSO sends the desired power profile to a community of prosumers that, in cooperative and competitive ways, modify their power consumption or production in order to offer the desired flexibility with the best price for the DSO and the maximum revenue for all prosumers. Such a virtual environment is the ideal platform for running smart contracts for the optimal management of the prosumers' flexibility and the blockchain could be used for assuring transparency to the process and avoiding controversy among the members of the community. In [44] the authors propose a decentralized price-based DR system taking into account the willingness of the prosumer to provide its flexibility to the grid. P2P aggregation for providing ancillary services is based on the assumption that prosumers selforganize and cooperate to effectively complete the assigned task. However, concerns about own resources consumption can limit this cooperation and invalidate the aggregation process [45]. For this reason, in [45] the authors propose that a suitable remunerative incentive mechanism must be implemented for encouraging secure participation of prosumers in a dynamic and collaborative P2P aggregation environment. In this sense, the Blockchain technology can be used for incentivizing prosumers through cryptocurrency and Bitcoin. The authors propose also a validation method against miners and collusion and a pricing strategy integrated into the incentive. The idea of the paper is in line with current tendency in the remuneration of ancillary services. As outlined in [27], where blockchain technology is used for loads participation (aggregation and demand response), a smart contract detects users preferences and reliability in order to provide fair access to the aggregation supported by incentives. Transparency, in this case, is needed in order for anyone to assess whether the criteria used for remuneration are valid. Moreover, a transparent platform may support the creation of energy purchasing groups that can directly interface with the wholesale market.

In these applications, both P2P trading and DR-aggregation, another main challenge resides in using privacy-preserving data exchange under GDPR (European General Data Protection Regulation) and this can be addressed by blockchain technology. The block immutability contrasts with the right to be forgotten provided by GDPR. There are three possible approaches for tackling this inconsistency, (i) data are stored off-chain and their cryptographic hash on the blockchain; (ii) to store encrypted data on the blockchain, then destroy the key in case these data have to be forgotten; (iii) use cryptographic approaches for erasable blocks [48], [49] . The first two solutions have some drawbacks: in the first, data availability is not guaranteed, in the second case an attacker could have in the future enough computational power to break the encryption scheme. On the other hand, typically, large amounts of data are to be exchanged and stored especially in DR applications, and this issue can be handled more efficiently storing data off-chain and loading on the blockchain only the hash of the data. Nodes showing less storage capability will indeed only store the headers of the blocks, while the whole blocks are stored remotely. In the application proposed in [22], a credit-based scheme for secure payments in energy transactions is considered. However the peculiarities of power systems world are not fully addressed since the pivotal role of DSOs is not considered.

\subsection{Suitability of Applying the Blockchain to power systems}

Starting from the analysis in subsections 2.1 and 2.2, Table 2 synthesizes for each application, benefits and technical implications. (R2-1.5) 
Table 2. Main applications of blockchain to power systems. (R2-1.5)

\begin{tabular}{|c|c|c|c|c|c|c|}
\hline & \multicolumn{3}{|c|}{ Electrical energy trading } & \multicolumn{3}{|c|}{ Renewable Energy Certification and Demand Response tracing } \\
\hline Area & $\begin{array}{l}\text { P2P electricity sale in } \\
\text { microgrids } \\
{[28],[29],[30],[31],[41]}\end{array}$ & $\begin{array}{l}\text { Involvement of the DSO for } \\
\text { handling balancing issues at } \\
\text { distribution level during P2P } \\
\text { transactions } \\
{[32],[33],[34],[35],[36],[37]} \\
{[38]}\end{array}$ & $\begin{array}{l}\text { Electricity trading in } \\
\text { presence of large amount } \\
\text { of renewables and EES } \\
{[39],[40],[41]}\end{array}$ & $\begin{array}{l}\text { P2P decentralized } \\
\text { management of Demand } \\
\text { Response in the grid } \\
{[11],[16],[19],[27],[43],} \\
{[54],[60],[62],[73]}\end{array}$ & $\begin{array}{l}\text { Demand Response applications } \\
\text { and distributed resources } \\
\text { aggregation } \\
{[16],[19],[25],[27],[33],[42],} \\
{[47],[61]}\end{array}$ & $\begin{array}{l}\text { Incentive mechanisms } \\
\text { for prosumers in } \\
\text { Demand Response } \\
\text { schemes } \\
\text { [10], [19], [23], [24], } \\
\text { [36], [37], [44], [45], [46 }\end{array}$ \\
\hline Benefits & $\begin{array}{l}\text { Improved security and } \\
\text { transparency } \\
\text { Best match between } \\
\text { demand and offers }\end{array}$ & $\begin{array}{l}\text { Improved security and } \\
\text { transparency } \\
\text { Reduced costs for the absence } \\
\text { of the Balancing Service } \\
\text { Provider (BSP) }\end{array}$ & $\begin{array}{l}\text { Improved security and } \\
\text { transparency } \\
\text { Maximization of the } \\
\text { energy produced by RES }\end{array}$ & $\begin{array}{l}\text { Improved security } \\
\text { Reduced costs for the } \\
\text { absence of the Balancing } \\
\text { Service Provider (BSP) }\end{array}$ & $\begin{array}{l}\text { Improved security and } \\
\text { transparency } \\
\text { Reduced costs for the absence } \\
\text { of the Balancing Service } \\
\text { Provider (BSP) } \\
\text { Avoid controversy among the } \\
\text { members of the community } \\
\text { Evaluation of prosumers' } \\
\text { reliability }\end{array}$ & $\begin{array}{l}\text { Improved transparenc } \\
\text { Privacy preservation }\end{array}$ \\
\hline Implications & $\begin{array}{l}\text { Reliability of all } \\
\text { apparatus certifying } \\
\text { measurements and } \\
\text { operations }\end{array}$ & $\begin{array}{l}\text { The DSO must assume a } \\
\text { "certifier" role for verifying } \\
\text { the technical feasibility of } \\
\text { transactions. }\end{array}$ & $\begin{array}{l}\text { Technical and balancing } \\
\text { issues must be taken into } \\
\text { account }\end{array}$ & $\begin{array}{l}\text { Absence of the BSP } \\
\text { Need for taking into } \\
\text { account energy losses and } \\
\text { technical constraints of the } \\
\text { grid. }\end{array}$ & $\begin{array}{l}\text { Absence of the BSP } \\
\text { A suitable remunerative } \\
\text { incentive mechanism must be } \\
\text { implemented for encouraging } \\
\text { secure participation of the } \\
\text { prosumer }\end{array}$ & $\begin{array}{l}\text { Creation of energy } \\
\text { purchasing groups tha } \\
\text { can directly interface } \\
\text { with the wholesale } \\
\text { market }\end{array}$ \\
\hline
\end{tabular}


Nevertheless, as highlighted in the introduction, one of the main problems to face is that to establish if the application of the blockchain technology to a specific issue of power systems' operation is suitable or not. A recent paper has discussed in general suitability of the application of the blockchain technology to a specific issue, providing a simple flow-chart to be used for this purpose [8],[50]. The flow chart is reported in Figure 2, with some modifications introduced for blockchain applications to power systems. Trying to understand the real need of blockchain technology for the electricity sector, both in P2P electricity trading, such as in certification and tracing for aggregation, such as it happens in Demand Response related applications, the issue is certainly multi-party. Smart meters and actuators at the prosumers' premises are decentralized sources of data, for which it is required to have the whole transactions history especially for assessing offers and demands along time and for quantifying remunerations (e.g. in Demand Response applications). Finally, time constraints for blockchain can either be strict or not in these applications because meters currently register energy in given time intervals and it is always possible to extract synthetic indicators for any profile. Additionally, in these cases, there is a need for transparency from all the involved parties, generally to overcome the traditional knowledge asymmetry between the DSO/energy retailer and customers. Transparency is a key feature especially for public and regulatory institutions, and it is generally desirable also for private companies. Indeed, it is worth to notice that, very often, DSOs and TSOs are involved into legal disputes with aggregators, prosumers, consumers and energy retailers due to various reasons (theft of electricity, excessive voltage drops, loss of service continuity, damages to the grid due to faults at the user's installation, etc.). In many cases, thanks to its characteristics of immutability, confidence and transparency, the blockchain could provide a quick solution to many disputes. This could modify the process described in [8] reinforcing the main role of transparency and immutability in the choice of the blockchain solution. Indeed, Figure 2 shows that, without a trusted authority, if transparency or immutability are required, the use of blockchain is always suggested. Full transparency, however, is needed towards pricing mechanisms and especially in the communication channel connecting DSO/aggregator/energy retailers and each consumer. Such a communication channel can even be invisible or partly visible to other consumers. In this case, the requirements for performance can lead to different types of blockchain. The classification depends on the access rules to the ledger and the permission to write it. The blockchain can be public or private depending on the access to the ledger, whereas it is named permissioned or permissionless depending on who is authorized to maintain the ledger [51]. Moreover, with respect to the proposal in [5] and [8], the flow chart is slightly modified including also the answer is not "Partly", due to the many different features of cyber-physical problems, such as power systems applications. 


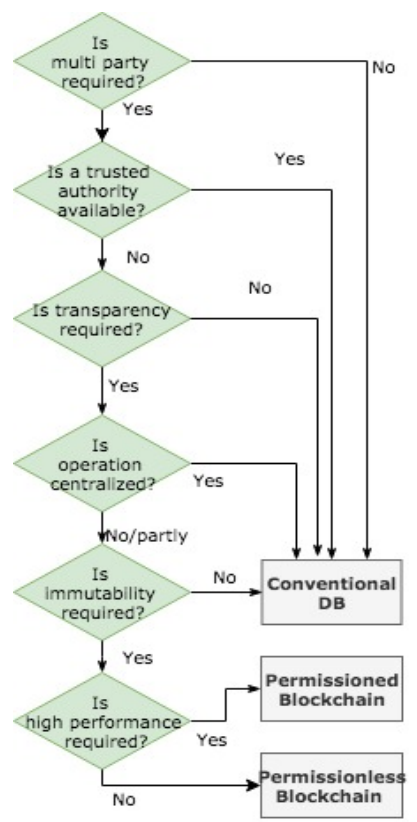

Figure 2. Suitability evaluation framework proposed by [8] adapted for power systems applications.

The power system sector involves several authorities such as Grid operators (TSOs and DSOs), Aggregators, Banks, Certification entities for physical apparatus providing data (IoT), Data Protection Officers (under General Data Protection Regulation, GDPR, 2018). Despite these authorities do not trust each other, the technical authorization for an energy transaction is always given by the grid operator, while financial assets of the parties as well as their identities are guaranteed by the blockchain itself under national regulation. Data Protection Officers instead call for the need for a centralized administration of the data system, thus indicating permissioned and private blockchain infrastructures. Operation control can only partly be decentralized since some technical issues are managed at central level (i.e.: balancing). When high performance, namely, scalability is required either security or decentralization must be limited, according to the blockchain trilemma [52] assumption, thus again pointing at permissioned or private blockchain technologies. The blockchain trilemma states that a blockchain system can provide at most two of the following three properties :

- Maximal decentralization of block production;

- Network security (its resistance to attacks);

- Scalability of the network (its ability to support load growth, related to the number of transactions that the network can process in a given time interval).

Decentralization refers to a low computational burden for any node of the blockchain, meaning the possibility even for a low computational power unit to fully take part in the blockchain community. Security is the property for which even in the presence of malicious attacks, none of the contents of 
the blocks could be altered or other malicious actions are prevented (e.g. the double spending of utility tokens).

Finally, Scalability is related to the possibility to increase the speed of transactions, namely the number of transactions that can be validated and agreed upon per second. This dimension concerns the efficiency of the technology in validating and 'finding a consensus' (see figure 1) on transactions contained in a block. Therefore, with respect to this item, both the technical validation if needed and the consensus algorithm have particular relevance.

The section below provides more details about permissioned versus permissionless blockchains.

\section{Blockchain technical requirements}

\subsection{General considerations}

There is a basic difference between blockchain applications that only manage digital assets and applications that interface with physical objects. This is also the case of the electrical energy world, where physical electricity transactions must be managed through smart meters and actuators [8]. In this case, else than a cryptographic signature or a digital recognition of a virtual item, there must be a similar 'certification' for a physical measurement or control system, as well as for the software controlling the actuators or other operations in the virtual dimension. Indeed, in the physical world, also complex applications (smart contracts) are needed that may provide a set of execution commands permitting to adhere to predefined workflows. This revolution was started since the inception of Ethereum [21], an open software platform based on blockchain technology that enables developers to build and deploy decentralized applications executed after a triggering event.

In the case of P2P trading applications, see Figure 3, the triggering event is connected both to availability of money, from one side, and to the availability of energy in the considered timeframe, from the other. Actually, in the electrical energy field, these conditions are necessary but not sufficient for any transaction to take place. There are some technical requirements connected to the capacity of connections and regulatory issues that must be considered. Such conditions cannot be verified in a distributed fashion in most cases and this calls for the action of Distribution System Operators, whose role becomes fundamental to ensure efficient integration of this technology in the grid. For this reason, blockchains [21] with some form of centralized control (called permissioned) may be more adequate to physical world applications especially in the power systems area where physical objects belonging to a third party are involved in the transaction. In these blockchains, only a few 'permissioned' nodes can create and write blocks. These nodes are not anonymous and they may implement different functions. With respect to this issue, it is advisable that, as an example, one of the blockchain nodes is from the DSO that supervises transactions and provides a certification about their technical feasibility [38]. This happens through a 'validation' procedure that can take place between steps 2 and 3 in figure 2. As an example, if validation of electricity transactions goes through the running of an Optimal Power Flow or similar procedure for which electrical data must be known, only the DSO can currently run it. Another challenge is about using the blockchain for aggregation purposes and for balancing. As an example, the NEMoGrid European project [53] involving various companies and research institutes from Germany, Switzerland and Sweden examines the economic and technical impact of electricity trade between households within an area, i.e. when selling solar power generated locally to other households. In this way, an insight into the mechanism of price variation in the presence of a P2P business model is gained as well as the suitability of blockchain technology to support flexibility and grid stability issues through aggregation in small pilot regions.

\subsection{Blockchain platforms}

Following the above introduction, this section explores the suitability of various blockchain platforms for different applications in the electrical energy sector. The focus will be on the 
following four technologies: Ethereum [54], Multichain 2.0 [55], Tendermint [56] and Hyperledger Fabric [57].

In the electricity sector, most applications deal with the emission of utility tokens. These are digital tokens of cryptocurrency that are issued in order to fund the development of the cryptocurrency and that can be later used to purchase a good or service offered by the issuer of the cryptocurrency. As a consequence they prove the ownership of an asset/privilege. In the applications here dealt with, these tokens can be exchanged (for example) as a counterparty of (green) electricity generation.

So, in this case, if one party generates green electricity is remunerated by a logic that is shared on the blockchain (defined as a smart contract) with a utility token. The latter can then be exchanged with security tokens also called securities, like bitcoin, storing a value.

Else than the features related to the Blockchain Trilemma [52] and recalled in section 2.3, another important feature is the flexibility in supporting smart contracts or other forms of logic. Indeed the possibility to implement smart contracts and not only 'local' client applications that do not change the state of the blockchain is a very relevant feature for blockchain platforms. Smart contracts must be able to process digital transactions and can be used to guarantee that a specific workflow is met. In all the applications concerning power systems, there is a need for smart contracts. In all cases, where a technical verification over the infrastructure is needed, the smart contract needs to run power analysis or power optimization codes. As an alternative, the smart contract can check some metadata sent as inputs of the smart contract. The difference is that in the first case all nodes run the smart contract to verify the transaction validity, while in the second case there is a trusted authority that must issue a 'permission', used under the form of metadata.

The mechanism of smart contract execution, delivering e-tokens if given flexibility at node A is provided, is depicted in Figure 3.

The output of the smart contract is the feasibility of the transaction and thus the consensus about starting the actuation of devices and the transfer of digital assets.

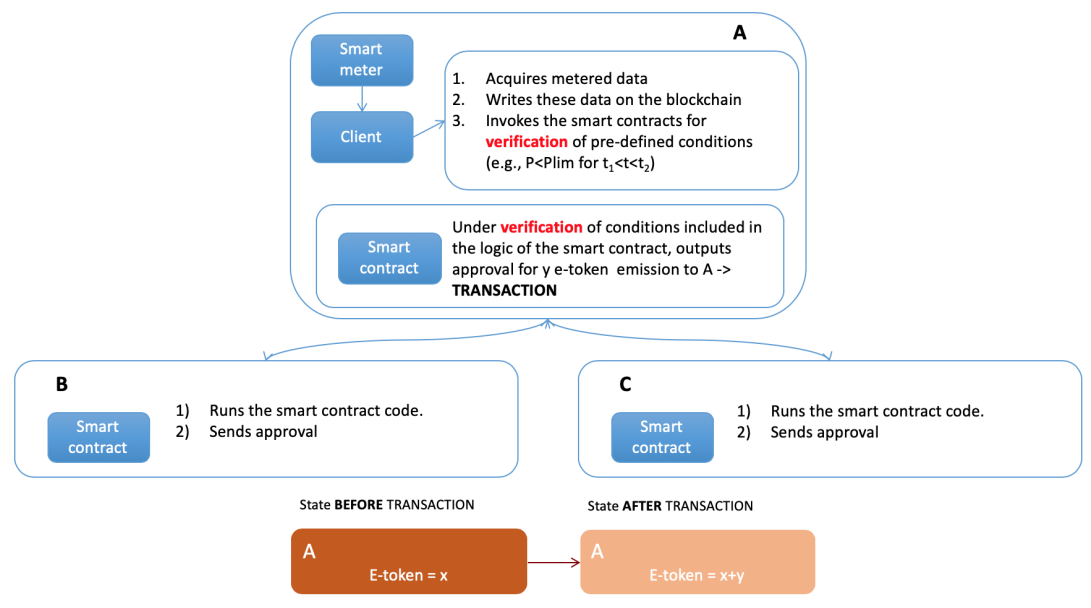

Figure 3. State changing as an effect of the execution of a smart contract.

3.3. Blockchain classification: Consensus algorithms

It is well known that consensus is one of the basic features for blockchain implementation. This mechanism allows to validate the blocks and insert them into the blockchain. The blockchain can be 
public or private depending on the access to the ledger, whereas it is named permissioned or permissionless depending on who is authorized to maintain the ledger [51].

Under the Proof of Work (PoW) mechanism, the node inserting the block is the one that solves a computationally-intensive problem before the others. As an outcome of this effort, it will be remunerated in crypto coins to pay for the energy consumed during calculations. Anyone can be a network node and can theoretically perform validations and blocks insertions. The greater the computing power the greater the probability of validating a block. The energy consumption becomes unbearable as the number of nodes scales up at the cost of high security. Differently from that, with proof of stake mechanism, the richer node will be more likely to validate the block. In case it has inserted a block which is invalid it will lose all of its stakes, however, for each block validated and inserted the node gets transaction fees.

The Proof of Stake (PoS) is a much less energy-consuming consensus mechanism than PoW. The creator of the next block is chosen via various combinations of random selection and a value considered as a stake hold by validators. In case the resources are shared unevenly among validators, PoS can introduce undesirable centralization. The set of validators propose and vote on the next block, and the weight of each validator's vote depends on the size of its deposit (i.e. stake). There are two major types of PoS: the chain-based PoS and the Byzantine Fault Tolerant (BFT) PoS. In the first type, a validator is selected pseudo-randomically at each time slot, then it gets the right to link a block to the longest chain. In the second type validators are randomly selected to propose blocks, then it starts a multi-round voting process that defines which block will be actually written on the blockchain.

The Proof of Authority $(\mathrm{PoA})$ is a reputation-based consensus algorithm where the validators of blocks are known with their real identities and are selected by their own reputation. There is a limited number of validators, which are arbitrarily selected as trustworthy entities. Despite this mechanism is more efficient than PoW and PoS, it appears to shift back to centralized trust, in validators. Therefore PoA focuses on security and performance but lacks the distributed dimension of the blockchain trilemma.

Since PoW is a thousand times more energy-consuming than PoS, it is important to understand when such a consensus mechanism is really needed, and this happens generally in public blockchain that is exposed to the Sybil attack. In most applications in the energy sector, permissioned blockchain technologies are adopted. As an example, [22] provides a unified energy blockchain based on consortium (or permissioned) blockchain for secure energy trading in various typical scenarios such as microgrids, energy harvesting networks, and vehicle-to-grids management. An innovative credit-based payment scheme allows reducing transaction confirmation delays as compared to traditional PoW blockchain. While Ethereum was originally created with a Proof of Work mechanism, it is now heading, by the release of Casper [58], to a lighter consensus mechanism. Tendermint provides a flexible and modifiable consensus mechanism: validators, that are the consensus participants, in turn, propose a block containing transactions, which can either be inserted into the blockchain only if more than $2 / 3$ of the validators execute the pre-commit ${ }^{1}$ of the same block in the same round. If this does not happen, the block passes to the next round [56]. Tendermint as lighter consensus mechanism can work with other smart contract platforms, such as an Ethereum Virtual Machine, EVM. In Hyperledger Fabric the consensus process is divided into 3 phases: Approval, Ordering and Validation. There are two main types of consensus: permissioned voting-based consensus or lottery-based consensus. Votingbased algorithms are advantageous as they provide low-latency purposes. When the majority of nodes validate a transaction or block, consent exists and the purpose occurs. Because voting-based

${ }^{1}$ The pre-commit is a voting mechanism performed by the validators to determine whether or not to insert the new block in the blockchain. 
algorithms typically require nodes to transfer messages to each of the other nodes on the network, the more nodes are present on the network, the longer it takes to reach consensus. This translates into a compromise between scalability and speed. Fabric can use consensus protocols that do not require a native cryptocurrency to incentivize expensive mining activities or to fuel an intelligent execution of contracts. The elimination of a cryptocurrency reduces some important risk/attack vectors and the absence of cryptographic mining operations means that the platform can be distributed at the same operating cost as any other distributed system [57]. In MultiChain, block validation is performed by identifiable nodes and there is a constraint on the number of blocks that can be created by the same miner within a given window. MultiChain implements this scheme using a parameter called mining diversity, which is constrained by $0 \leq$ mining diversity $\leq 1$. At each round a validator is extracted, whose probability of being extracted depends on the mining diversity. The extraction process must be performed by diversified groups of nodes that are allowed to do so. The permission does not only concern the extraction of data and blocks but also the sending/receiving of transactions and the display of the content of blocks. A block is considered valid only if the miner that was extracted to validate the current block did not validate the previous block. This imposes a round-robin program, in which authorized miners must create rolling blocks to generate a valid blockchain [55].

\subsection{Smart contracts implementation in power systems applications}

As already outlined, an important issue of blockchain technologies applied to power applications concerns the way in which smart contracts can be implemented. More in detail, when Alice wants to sell electricity to Bob three smart contracts need to be implemented:

1) awarding Alice with e-tokens (utility tokens) corresponding to the power injected in the grid;

2) awarding Bob with e-tokens (utility tokens) corresponding to the crypto assets (security tokens) transferred to Alice (Ether or other cryptocurrencies);

3) destroying e-tokens (utility tokens) as Bob consumes electricity.

The first kind is implemented for certification and tracing applications. All are implemented for trading.

Ethereum was created for the purpose of running smart contracts. The coding language is 'Solidity'. The 'Solidity' language, although Turing complete, shows some shortcomings that do not make it suitable for implementing complex coding, such as it would happen for Load flow, Optimal Power Flow computations or certain optimization codes. Also, it has size limits on the input data it can accept. It usually works on a relevant database of states which is a subset of data taken from the blockchain and that is updated as the smart contract is executed. Figure 4 shows the way in which Ethereum smart contracts are implemented. 


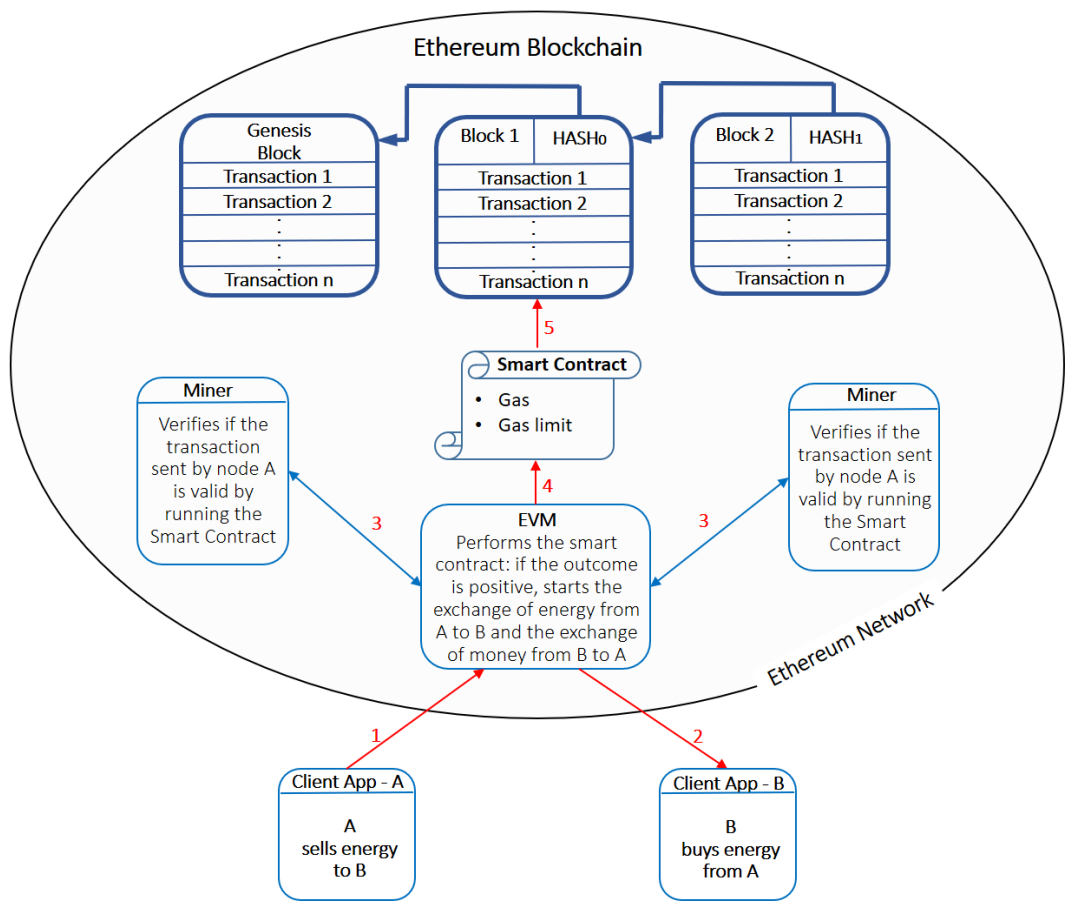

Figure 4. State changing as an effect of the execution of a smart contract under Ethereum.

Ethereum is a public blockchain that can run smart contracts; these are compiled in "bytecode" and are read and executed by a virtual machine called "Ethereum Virtual Machine" (EVM). Each node of the network has its own EVM which executes, in particular, the same set of instructions as the other nodes. The smart contracts, before being executed, are compiled producing the bytecode which can then be interpreted by the EVM. So, in a nutshell, the EVM is the environment where smart contracts are executed.

Multichain 2.0 instead, using the smart filtering technique, allows some sort of 'smart contract' implementation, although complex logic implementations are not possible. If a transaction is approved by the logic of the smart filter, with the required level of consensus, it is added to the blockchain. Transaction filters validate individual transactions without reference to any global state. Of course, to be comparable to smart contracts, also smart filters need to run identically on every node in a blockchain, preventing from any source of non-determinism such as reading the time, using random numbers, accessing network or disk, or performing math operations that depend on the host server's architecture. The role of a filter is to return a boolean saying whether a transaction is acceptable. Multichain's API (Application Program Interface) includes commands for creating filters, testing them on previous or new transactions, and activating them subject to administrator consensus. Unlike smart contracts, if a bug is discovered in the code for a filter, it can easily be replaced by a new version. It can also be easily disabled if it enters an infinite loop.

Tendermint, see Figure 5, is a consensus engine that allows replicating the status of an application in all the machines that are part of the network. The peculiarity, compared to other blockchains, is that it allows developing a local application in any programming language [56]. Tendermint, 
however, does not allow to directly implement smart contracts. For this reason, as an example, the EVM can be employed to implement the smart contract and Tendermint can be employed as a consensus engine, see Figure 7. While in Ethereum, Figure 6, both the smart contract execution and the mining must be paid, here the compensation is only recognized for running the smart contract. The consensus algorithm is indeed in general lighter and, in this way, a private or permissioned blockchain can be implemented. Moreover a local client application can query data on the blockchain through the Application BlockChain Interface (ABCI), which is a tool that allows the user application, external to the blockchain and writable in any programming language, to communicate with the blockchain.

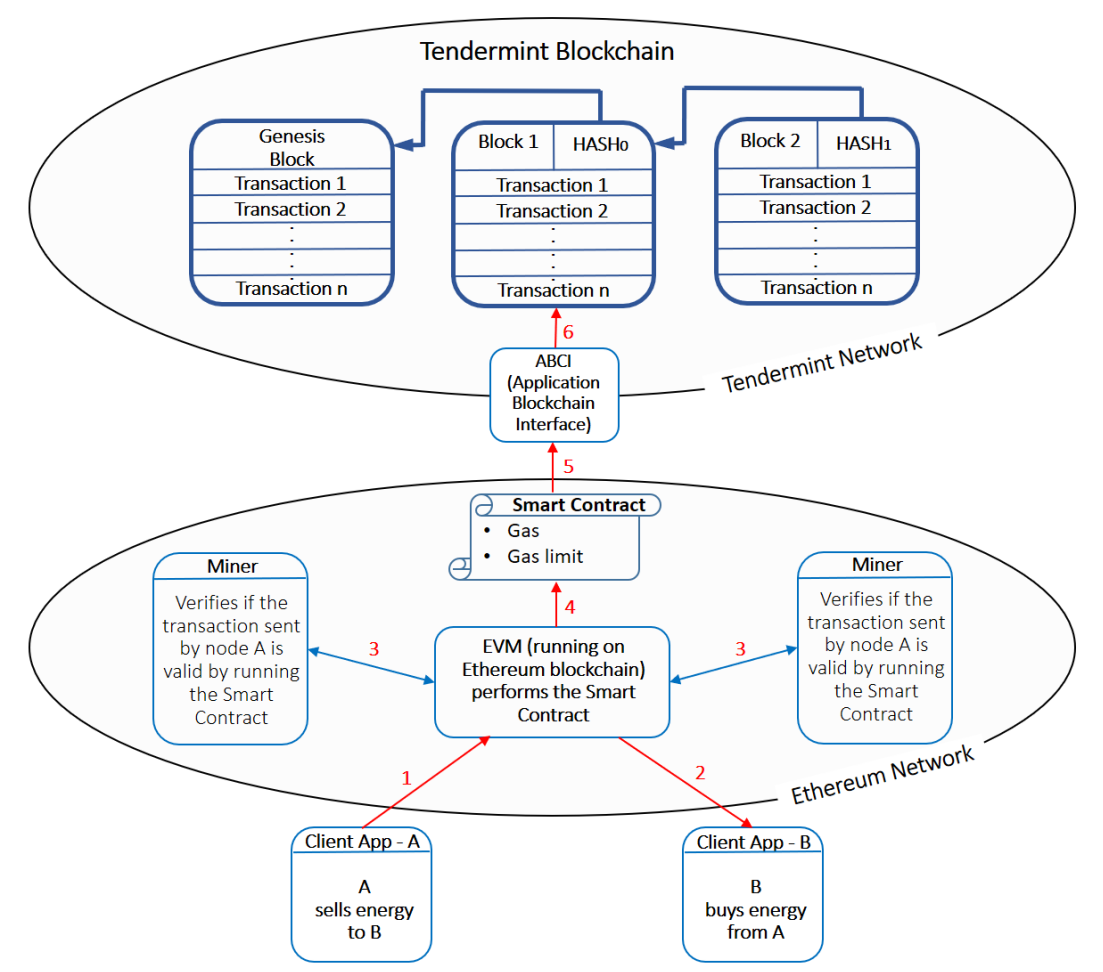

Figure 5. State changing as an effect of the execution of a smart contract under Tendermint and EVM.

Hyperledger Fabric is a platform designed for use in business contexts, which offers some key differentiation features compared to other popular general ledger or blockchain platforms. Fabric has a highly modular and configurable architecture that enables innovation, versatility and optimization for a wide range of use cases in the industry. Fabric, unlike Ethereum, supports smart contracts written in generic programming languages like Java, Go and Node.js. The Fabric platform is also permissioned, which means that, unlike a public network without authorization, the participants are known to each other rather than anonymous and therefore completely unreliable. In Fabric, the smart contracts are called "chaincodes", they work as a reliable distributed application that acquires its security/trust from the blockchain and the underlying consensus among the peers. Most existing blockchain platforms with smart-contracts following an order-execute architecture in which the consensus protocol validates, orders and disseminates transactions to all peers. Smart 
contracts executed in a blockchain that operates with the order-execute architecture must be deterministic; otherwise, consensus may never be reached. To address the problem of nondeterminism, many platforms require smart contracts to be written in a non-standard or domainspecific language (such as Solidity in Ethereum) so that non-deterministic operations can be eliminated. Fabric introduces a new architecture for the transactions called execute-order-validate, whose description is out of the scope of this paper. Details can be found in [57]. In an Hyperledger Fabric network, the logic of the transaction and its validity is established by the smart contract, which is executed by one of the peers participating in the network when a client sends a request of transaction. The chaincode is a tool that allows the user to interact with the ledger, in fact any operation, even a simple query, is performed through the smart contract. If the transaction sent by the user is consistent with the logic implemented by the smart contract, the new transaction in sent to the "orderer node", that is the component responsible for the consensus process. Transactions must be written to the ledger in the order in which they occur. In order for this to happen, it is necessary to establish the order of the transactions and create a method to reject the erroneous transactions that were entered in the ledger by mistake (or maliciously). This task in hyperledger fabric is entrusted to the orderer node. The consensus algorithm is quite similar to the one needed in Tendermint, but in this case the algorithm is executed by the orderer nodes. In a hyperledger fabric network, the communication among the various components takes place through the "channels", communication mechanisms which allows for data isolation and confidentiality and by which users can communicate with each other. The whole process to record a new transaction and all the components of the network are shown in Figure 6. On a specific channel, the ledger and the specific smart contract is shared across the peers in that channel, and transacting parties must be properly authenticated to a channel in order to interact with it. The channels are the tools that allow privacy within the network and at the same time transparency on the smart contract execution, since all peers on the same channel have a copy of the same smart contract. Hyperledger Fabric also offers the possibility of creating different channels on the same network, allowing a group of participants to create a separate transaction ledger. This is a particularly important option for networks in which some participants may be competitors and do not want every transaction made by them to be known to all participants. If two participants form a channel, these two participants, and no one else, have copies of the ledger for that channel preserving the privacy and confidentiality of both. More information about the network structure can be [59]. 


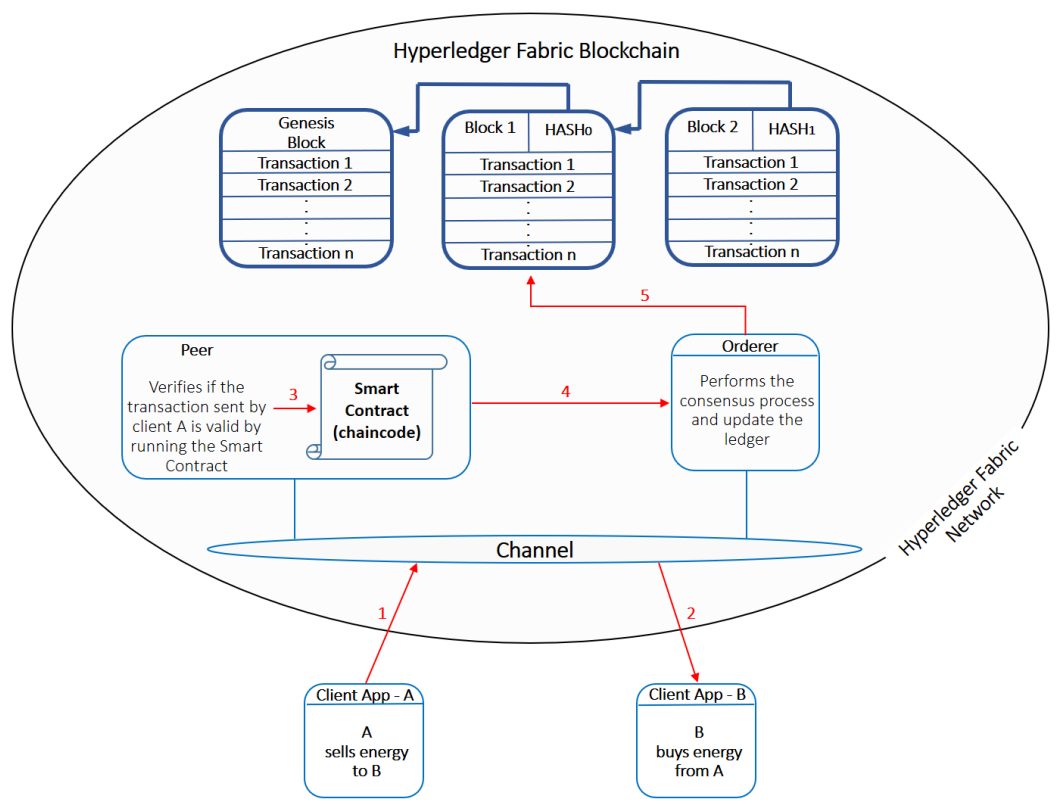

Figure 6. State changing after the execution of a smart contract under Hyperledger.

With reference to figure 2, compared to the other blockchain technologies, the fabric blockchain seems to be the most suitable for power system applications, as it allows easy implementation due to the possibility of using standard programming languages for smart contracts, transparency because the smart contract is shared with all users linked on the same channel and high performance because it is a private network.

In what follows a comparison of the four platforms on some relevant features is proposed.

Unlike other platforms, MultiChain has some built-in abstractions providing basic building blocks for blockchain-driven applications, which are assets and streams. Three are the areas covered by these abstractions: (a) dynamic permissions, (b) transferable assets, and (c) data storage [52]. Another important issue is the possibility to create any number of "streams" on a chain, which essentially is a way to publish, index and retrieve both on-chain or off-chain data in different formats: JSON, text or binary. In this way, when statistical analysis or any other learning technique must use as input data from the blockchain, a suitable arrangement of data may turn to be useful.

Unlike other platforms, Multichain voluntarily limits the capabilities of its smart filters, which are not as flexible as Fabric chaincodes and Ethereum smart contracts. The positive side-effect is that while a but in the on-chain logic of other platforms can be destructive, a bug on a smart filter has limited impact, can be easily corrected by deactivating the filter and replacing it with a newer version.

As an example, for loads aggregation, it may be useful to classify users based on their electrical proximity depending on the network asset. This can help to support different functions to the grid and depends on data that are not necessarily on the blockchain and can dynamically change. In the same way, permissions to trade electricity may be inhibited to customers who do not behave well in terms of payments or quality of service delivered to other peers. 
Another important feature is determinism. Since smart contracts are run by several machines it must be guaranteed that they all give the same result. If this does not happen, a 'fork' can take place and the blockchain can show different states to different machines for some time. Causes of non-determinism in smart contracts execution are the reliance on external documents, the use of random number generators or even access to local time.

In the case of Ethereum, Solidity cannot encode any non-deterministic operation. Also Tendermint, when relies on EVM, has the same behavior. While in Multichain, determinism is obtained through a restricted version of Javascript with randomicity disabled that is used for implementing smart filters. The latter issue is conflict prevention. How does each technology deal with the possibility that two transactions, each of which is valid, do not conflict with each other? In the simplest example, imagine that Alice has $\$ 10$ in a P2P power transaction ledger and broadcasts two transactions - one sending $\$ 8$ to Bob to buy electricity, and the other sending $\$ 7$ to Charlie to buy electricity. Clearly, only one of these transactions can be allowed to succeed. If Multichain 2.0 is running, the transaction single-spend rule will prevent the chronologically second transaction to take place. In Ethereum transaction conflicts such as Alice's two payments are not immediately visible to the engine. They are instead detected by the smart contract processing the transactions. When processing each of Alice's payments, the smart contract verifies whether her balance is enough. While in the first case, the double-spending is simply not possible, in the second case, the verification is 'a posteriori' and a delay for finding it out is needed. All these considerations are summarized in Table 3.

Table 3. Features of Ethereum, Tendermint and Multichain on smart contracts execution.

\begin{tabular}{|l|l|l|l|l|}
\hline \multicolumn{1}{|c|}{ Ethereum } & Tendermint & Multichain & Hyperledger Fabric \\
\hline Consensus & $\begin{array}{l}\text { PoW, PoS } \\
\text { (Casper) }\end{array}$ & $\begin{array}{l}\text { Voting } \\
\text { mechanism }\end{array}$ & $\begin{array}{l}\text { Based on mining } \\
\text { diversity }\end{array}$ & $\begin{array}{l}\text { Voting or Lottery } \\
\text { mechanism }\end{array}$ \\
\hline $\begin{array}{l}\text { Smart contract } \\
\text { coding }\end{array}$ & Solidity & $\begin{array}{l}\text { Solidity (if uses } \\
\text { EVM) }\end{array}$ & Javascript & Java, Go, Javascript \\
\hline Built-in rules & No & No & Yes & Yes \\
\hline $\begin{array}{l}\text { Dynamic } \\
\text { permissions }\end{array}$ & No & No & Yes & No \\
\hline $\begin{array}{l}\text { Conflict } \\
\text { prevention }\end{array}$ & $\begin{array}{l}\text { Contract } \\
\text { checks }\end{array}$ & Contract checks & $\begin{array}{l}\text { Single-spend } \\
\text { rule }\end{array}$ & Single-spend rule \\
\hline $\begin{array}{l}\text { Speed in conflict } \\
\text { management }\end{array}$ & $\begin{array}{l}\text { Around 10 s } \\
\text { (confirmation) }\end{array}$ & $\begin{array}{l}\text { Around 10 s } \\
\text { (confirmation) }\end{array}$ & $\begin{array}{l}\text { Around 1 s } \\
\text { (propagation) }\end{array}$ & $\begin{array}{l}\text { Around 1 s } \\
\text { (propagation) }\end{array}$ \\
\hline Smart contracts & Contract & $\begin{array}{l}\text { Uses EVM (for } \\
\text { example) }\end{array}$ & Smart filters & Chaincodes \\
\hline
\end{tabular}

In the power systems area, the third parties are the grid operators and the grid balancing authorities. There are a few basic issues that cannot be neglected:

- Electrical grid data are not publicly available

- Permission to connect to the grid is given by grid operators

- Power Flow analysis codes are the solution of highly nonlinear systems

- Optimal Power Flow codes have highly non-linear optimization problems.

A number of smart contracts in electricity trading in the literature do not neglect the technical features. 
Referring to the classification of applications proposed, they all apply, but in some cases, i.e. Demand Response and certification (tracing), the technical verification about feasibility of a given injection is typically made in advance and the injection/consumption follows a precise technical request from the aggregator/DSO and in turn from the TSO/energy trader. In other applications, such as P2P electricity trading, it may happen that smart contracts need to integrate Optimal Power Flow codes or Power flow analysis. This impacts the complexity of smart contracts that may or may not account for technical feasibility when the approval to the transaction is issued.

In all cases, if a public blockchain is used for P2P trading, complex algorithms accessing electrical grid data and other customers loads data may be run as smart contracts. In [34], each transaction fee between two nodes is calculated based on losses calculation and power flows tracing. In order to do so, a load flow and a power flow tracing algorithm must be run. The power losses term thus varies along time, due to the superposition of transactions and the consequent change of status of the electrical network. The authors consider the DSO as third party validating transactions. In [60], the authors again formulate the trading problem as a grid constrained problem, with OPF solution and sensitivity coefficients calculation. The authors still rely on a third party (DSO) validation of each transaction. Other papers [61], [62] proposing the Alternating Direction Method of Multipliers, ADMM, an algorithm for running decentralized Optimal Power Flow, set the aggregation step of results to a smart contract. In this case, however, the authors consider that grid data are publicly available. Due to the limited complexity of the smart contract code, it was implemented on an Ethereum platform. However the authors recognize that the ADMM communication overhead limits its use for day ahead scheduling. As a result, for P2P trading applications, where network constraints must be checked, a permissioned blockchain with required running of a client application at the premise of a validator DSO node is needed. In this case, either Multichain 2.0 with smart filtering or Tendermint with light consensus algorithm and EVM for smart contracts execution [63] may be a suitable choice. Then, for P2P trading applications, where network constraints are not checked, a public blockchain like Ethereum can be used. Finally, when it is required flexibility in smart contracts and privacy is a valuable factor, as in the case of measurements taken by energy meters, it is convenient to use Hyperledger Fabric and its channels.

\section{Some examples of Blockchain experiences in power systems}

The Austrian company Wien Energie, the largest supplier of energy in Austria, is testing a range of blockchain use cases in anticipation of rolling out a vast range of services that might soon incorporate them. The idea from the Chief Innovation Officer is that is the technology is really disruptive for P2P trading they do not want to be left behind. The blockchain-based trading platform is proprietary and was developed by a Canadian start-up IBL [64]. Austria, however, was already at the forefront of B2B integration in the energy field with the Energy Data Exchange Austria system [65]. Another large project in Europe is Enerchain involving 42 entities (May 2018). It chose Tendermint as the current underlying blockchain (specifically "consensus") technology. Among those who take part in the initiative, there are E.On, Total, Wien Energie, EDF and Vattenfall. Their aim is to implement a decentralized European marketplace for energy trading. A different pilot project is going on in the Netherlands with the Vandbebron company. The latter is providing TenneT (dutch and german TSO) with flexible capacities from a coordinated pool of electric vehicles and recharging points. In a second pilot project in Germany, the German company Sonnen is providing TenneT with flexible capacities from home storage systems that are interconnected. The Sonnen e-services control center connects home storage systems and adjusts charging operation according to the main grid condition [66]. Still in Germany Conjoule is developing a platform for peer-to-peer electricity trading. Customers will be able to sell and buy electricity from neighbors through a platform that will provide track and trace functionalities and then transact energy. 
The Brooklyn Microgrid project in the US [63] relies on a private blockchain to manage the P2P electricity exchanges between neighbors in a New Your district. Market algorithms are being implemented to establish dynamic pricing but also provide ancillary services to the grid. Grid+ is based in the United States. It delivers a product called 'Grid + ' which purchases/sells electricity every fifteen minutes acting on different markets. Blockchain is here used to speed peer-to-peer transactions bringing renewable energy and storage units into the marketplace. They are currently based in Texas, and currently involves thousand of consumers. They accept to buy a number of GRID tokens that are then used by the electronic sales agent to bargain electricity. Tokyo Electric Power Company, a Japanese utility, has recently signed a Memorandum of Understanding with Grid+ and is supposed to generate Pacific region market exposure to the platform Grid+. Alastria in Spain is the first national non-profit consortium that is providing a multi-purpose collaborative platform focusing on many areas of commerce including energy. The aim of this consortium is to speed up the digital transformation of business as well as of industry on which consortium participants can generate digital tokens supporting new products and services development.

Solara blockchain is a different system. It was created to incentivize solar energy generation. Each owner of a solar generation asset can register to the Solara platform and receive some crypto coins as a reward. These coins can be used to reward miners or to provide stake under a number of consensus algorithm. For this reason, the mechanism incentivizes solar energy production on one hand and the blockchain technology diffusion on the other. Solara also provides hardware and software for tracing of electricity, by adding to each piece of solar source (panel) or battery unit a chip with a unique identity. In this way, electricity generation is fully traceable with a mechanism that directly writes data over the blockchain. Solara indeed introduces the so-called proof of Fusion by a cryptographic signature, namely a certified "witness of the output of the panel at the point of production and at the time of production, and feeds it directly and securely into the local SOLARA Edge Blockchain Node" [67]. In this application, the problem of physical assets data input for running smart contracts is dealt with. Other projects do not involve end-users.

The companies E.On and Enel see the blockchain technology as an opportunity to reduce electricity costs for end-users not involving them directly into the blockchain platform. The first experimental trading was indeed carried out between the two global energy giants based on the Enerchain platform. Austrian startup Grid Singularity is developing a blockchain-based hierarchical decentralized energy exchange platform. The platform should host applications such as validating electricity trades, monitoring grid equipment, in this way, such a platform has the potential to prolong the life of equipments and increase large and small prosumers earnings. Table 4 summarizes the reported experiments with relevant features. Although other reports exist proposing a more extended review of existing applications, only a brief summary is here reported to focus on the most exemplary applications.

Table 4. Some of the existing blockchain applications to energy trading and features.

\begin{tabular}{|c|c|c|c|c|c|}
\hline Project & Time & $\begin{array}{c}\text { Energy } \\
\text { market }\end{array}$ & Commodities & Type & $\begin{array}{c}\text { Blockchain } \\
\text { technology }\end{array}$ \\
\hline $\begin{array}{c}\text { Enerchain } \\
\text { (EU) }\end{array}$ & $\begin{array}{c}\text { Intraday (45 } \\
\text { mins and hours); } \\
\text { D-A; weekly, } \\
\text { monthly, } \\
\text { quarterly and } \\
\text { yearly baseload }\end{array}$ & $\begin{array}{c}\text { Over The Counter } \\
\text { electricity } \\
\text { trading/P2P }\end{array}$ & $\begin{array}{c}\text { Gas and } \\
\text { electricity } \\
\text { ary smart contract } \\
\text { application (Ponton) }\end{array}$ \\
\hline $\begin{array}{c}\text { Viertel district } \\
\text { (Wien -Wien } \\
\text { Energie) }\end{array}$ & Balancing & $\begin{array}{c}\text { V2G/P2G } \\
\text { Retail }\end{array}$ & $\begin{array}{c}\text { Gas and } \\
\text { electricity }\end{array}$ & Private & Interbit BTL \\
\hline
\end{tabular}




\begin{tabular}{|c|c|c|c|c|c|}
\hline (AU) & & & & & \\
\hline $\begin{array}{c}\text { Tennet - } \\
\text { Vanderbron - } \\
\text { Sonnen (NL) }\end{array}$ & Balancing & $\begin{array}{l}\text { V2G/home- } \\
\text { storage2G }\end{array}$ & Electricity & Private (TSO) & Hyperledger \\
\hline $\begin{array}{l}\text { Electron } \\
\text { (UK) }\end{array}$ & $\begin{array}{l}\text { Balancing; } \\
\text { Meter services; } \\
\text { Community } \\
\text { energy projects }\end{array}$ & $\begin{array}{l}\mathrm{DR} / \mathrm{P} 2 \mathrm{P} / \text { flexibility } \\
\text { trading }\end{array}$ & Electricity & Permissioned & $\begin{array}{c}\text { Ecosystem of } \\
\text { platforms based on } \\
\text { Ethereum }\end{array}$ \\
\hline $\begin{array}{c}\text { Energy Web } \\
\text { Foundation (Grid } \\
\text { singularity and } \\
\text { Rocky mountain) } \\
\text { (US) }\end{array}$ & any & $\begin{array}{l}\text { Multi-purpose } \\
\text { Experimental } \\
\text { framework }\end{array}$ & Electricity & Permissioned & $\begin{array}{l}\text { Trading platforms } \\
\text { based on Ethereum }\end{array}$ \\
\hline $\begin{array}{l}\text { Conjoule } \\
\text { (DE) }\end{array}$ & Intraday & P2P & Electricity & Permissioned & $\begin{array}{l}\text { Self-developed } \\
\text { trading platform }\end{array}$ \\
\hline $\begin{array}{l}\text { Alastria } \\
\text { (ESP) }\end{array}$ & NA & $\begin{array}{l}\text { Multi-purpose } \\
\text { Experimental } \\
\text { framework }\end{array}$ & $\begin{array}{l}\text { Electricity and } \\
\text { other goods }\end{array}$ & Permissioned & $\begin{array}{l}\text { Quorum evolving to } \\
\text { Hyperledger }\end{array}$ \\
\hline $\begin{array}{l}\text { Grid+ } \\
\text { (US) }\end{array}$ & Any & P2P & Electricity & Permissioned & $\begin{array}{c}\text { Trading platform } \\
\text { based on Ethereum }\end{array}$ \\
\hline $\begin{array}{l}\text { Solara } \\
\text { (AUS) }\end{array}$ & l & $\begin{array}{l}\text { Tracing and } \\
\text { certification of } \\
\text { provenance/Fundr } \\
\text { aising through } \\
\text { ICO (Solarcoin) }\end{array}$ & Solar electricity & Permissioned & $\begin{array}{c}\text { Energy Web } \\
\text { Foundation } \\
\text { technology \& Self- } \\
\text { developed platform } \\
\text { (Lite version of } \\
\text { Bitcoin) }\end{array}$ \\
\hline $\begin{array}{c}\text { Green } \\
\text { Powerexchange } \\
\text { (BRB) }\end{array}$ & Intraday & P2P & Electricity & Public & Ethereum \\
\hline $\begin{array}{l}\text { The Brooklyn } \\
\text { Microgrid } \\
\text { (US) }\end{array}$ & Intraday & P2P & Electricity & Private & $\begin{array}{l}\text { Tendermint/Propriet } \\
\text { ary smart contract } \\
\text { application }\end{array}$ \\
\hline $\begin{array}{l}\text { Powerledger } \\
\text { (AUS) }\end{array}$ & Intraday & P2P/EVs & Electricity & Permissioned & $\begin{array}{l}\text { Trading platform } \\
\text { based on Ethereum }\end{array}$ \\
\hline
\end{tabular}

\section{Perspectives for future applications}

Most innovative applications of the blockchain to the electricity sector mostly concern the use of this technology for tracing and trading ancillary services provision and for implementing all levels of the energy market. In what follows the two aspects are analyzed and commented.

5.1. The blockchain technology for ancillary services provision 
In addition to peer-to-peer electricity transactions and Aggregation and Demand Response tracing, another application of blockchain technology in the energy sector could be the full management of network operations related to voltage and frequency regulation (ancillary services). With decentralization, the number of transactions to guarantee ancillary services will increase significantly as the level of uncertainty will increase as a consequence of deep penetration of RES. While for P2P trading the aim of enabling technologies like blockchain is to incentivize and simplify electricity trading between users by limiting third parties intervention, for ancillary services provision, the so-called third parties (grid operators and balancing responsible parties) themselves could trade it with prosumers. The prosumers have to be notified about the need for real or reactive power injection into the network. As a result, this service would lead to trade between end-users and grid operators, in order to improve the energy supply service. In this case, a hybrid blockchain, incorporating the features of both public and private networks, is needed. For this application, again a transparent and secure blockchain, in which all users can join, is needed but with a verification and consensus protocol controlled by a pre-established set of nodes (verification nodes). In this case indeed, such as in other cases already seen, the algorithm for deciding which node should provide what power at what time is an optimal power flow. The main potential of blockchain technology, in this scenario, is that it can provide a secure foundation for different markets that focus on ancillary services provision and, at the same time, reduce barriers to market entry for smaller devices connected to the network. Because electricity grids are critical infrastructures, there are many different rules and regulations to ensure that entities participating in ancillary services provision respect the contractual relationships they have with network operators [68]. Because of these complex regulations, small prosumers do not provide ancillary services, although they theoretically could. With blockchain technology, smart contracts could be used to apply the current ruling system for ancillary services into the digital world. Once smart contracts have been defined correctly, they can be used to facilitate market processes for the collection of ancillary services in a safe and efficient manner. Furthermore, these smart contracts could facilitate market processes for ancillary services provision from all users of the network (from large industrial consumers to home users). An example of this approach is the D3A framework developed by the Energy Web Foundation, which aims at the integration of decentralized assets up to different domestic devices in local and national markets for energy and ancillary services. WePower with the Estonian TSO Elering or Tennet with the battery storage company Sonnen are currently examining how smart contracts could be applied to perform tasks that help to balance the network. Thinking of a future scenario, network operations could become an increasingly digitized business with elements that traditional network operators could not provide. Thus, the government may choose to allocate network operations to a different entity that has the ability to manage digitized power grids more efficiently. This is a model that we already know from transmission grids in the United States, where the ownership of resources is of utilities, while the Independent System Operators (ISO) deal with network operations. Although the technological barriers are still many, such as the management and accounting of assets and the veracity of the measurement of the same assets, with the use of the blockchain it is possible to make a positive contribution to overcome them [69]. A further particular case that deserves a more detailed discussion is the possible application of the blockchain to the electricity market in Europe.

\subsection{The blockchain technology for the electricity market in Europe}

Time and thus scalability seems to be quite relevant when blockchain technology must be coupled to different electrical energy markets. In this section, a broad overview of how the electricity market is organized in Italy and in Europe is provided. The aim of this subsection is to understand how, in the current organization of the market, the blockchain-based energy trading could be used. The electricity market in Italy and in most European countries has changed a lot recently due to the transition to a low-carbon economy. Electricity is currently traded at wholesale level or at retail level. Trading takes place between generators, suppliers and traders (wholesale) or between suppliers and end-users (retail). Recently, however, else than these, new forms of energy trading 
are appearing. These are defined as $\mathrm{P} 2 \mathrm{P}$ markets. Considering the type of transactions, the electricity market can further be divided into regulated and non-regulated markets. The first type comprises spot and forward markets, while the second mostly comprises Over The Counter, OTC, markets, which typically are forward markets based on bilateral contracts. A large part of the exchanges into non regulated markets are devoted to purely financial transactions. The main problem with bilateral transactions that could be solved through the blockchain technology is the limited access from operators. The latter as an example, in the regulated markets in Italy, must be recognized by the GME. In this case, the scalability issue can be considered not as a primary need, since the exchanges are future deliveries. The wholesale electricity markets include both spot and forward market schemes. The main difference between the two is the way in which the bargain is carried out. In the first case (spot market), bargain is organized into market sessions with defined reference period of delivery, where each counterpart offers its capacity and price. The most relevant figure is the Day-Ahead Market (DAM) where the bargain is limited by the defined market sessions but price setting is hourly; in the forward market instead the bargain is continuous and in addition to price and volume also the delivery period is negotiated (futures). In both cases, bargain is carried out on virtual platforms (IPEX, EEX...) collecting offers and implementing the algorithm for clearing. In forward markets, supported by bilateral trading, a supplier and a consumer agree on a trade contract by directly interacting with each other. In this case, trading can take the market price published by the power exchange as reference price. In organized bilateral trading, market participants submit generation and demand bids to a market platform, which is cleared continuously; one market player can bilaterally accept the bid of another market player, resulting in different prices for each trade. While the so-called exchange markets (spot and forward) have as counterpart in Italy the GME, bilateral bargain is carried out between two parties and thus there is no central counterpart, but the physical delivery must always be accepted by the market manager (GME in Italy). This is why the outcomes of non regulated platforms for OTC must be notified to GME and finally to the TSO. A generic transaction on the futures market implies as main information: the parts in the contract, the amount of energy that is object of the exchange offered or to be purchased, the profile with which such capacity is offered (Baseload, peak, offpeak), and duration (month, three-months, year). The introduction of the blockchain technology to support wholesale markets, and in particular regulated and non regulated bilateral (OTC) markets [70], [4] can reduce the counterparty risk and improve transparency while keeping the privacy-preserving feature. The Enerchain project [71] is developing a blockchain-based P2P trading platform to complement, or replace, the wholesale electricity market improving transparency.

The retail market implies the presence of a wholesale buyer that then sells to individual customers. This is, for example, the case of aggregators. In this case, blockchain technology could improve transparency among consumers and between the TSO and the aggregator.

As the European Directive on common rules for the electricity markets claims, "integrated short term markets are missing", while these short-term electricity markets would allow "trading RES-E across borders" and are the "key for the successful integration of RES-E into the market". The same directive recognizes that "the advent of digitalization, internet-based metering and trading solutions enable industry, businesses and even households to generate and store electricity, as well as participate in electricity markets via so-called 'demand response' solutions. Besides, the electricity market of the next decade will be characterized by more variable and decentralized electricity production, an increased interdependence between Member States and new technological opportunities for consumers to reduce their bills and actively participate in electricity markets through demand response, self-consumption or storage" [72]. The directive also encourages local energy communities as a means for fostering renewable energy penetration. Such energy communities have similar features across Europe, however in some countries like in Italy, there are a few issues that make them lose competitiveness as compared to other forms of supply. In Italy, even if two private parties exchange electricity through a private network (Sistemi efficienti di utente) connected to the main grid, the buyer has to pay overhead taxes for network services, 
although these taxes are reduced as compared to standard tariffs. Still, it seems, that the so-called P2P markets [73], [74] are still in an experimental phase.

The work in [12] makes a wide overview of P2P markets. The paper outlines three different types: (i) full P2P market; (ii) community-based market; and (iii) hybrid P2P market. In the full P2P market, agents (i.e. producers, consumers and prosumers) interact without third parties (i.e.: retailer or market operator). A couple of agents can agree on a certain amount of energy exchanged for a given price without centralized supervision. In this case, the agents should be able to understand whether the transaction is technically feasible and to what extent it influences other nodes in the grid. The community-based market is more structured, it implies the presence of a third entity, namely a community manager, to rule transactions among agents inside the community. This entity also manages the interaction of the community with the main grid. The hybrid type is a combination of the two previous market structures, ending up with different layers for trading energy. In each layer, communities and single agents may interact directly. This third type is more organized than a P2P market but not as structured as a community-based market. P2P markets are the natural environment for blockchain technology and distributed ledgers. What appears evident is that while some wholesale markets (forward) do not account for technical feasibility of transactions that are successively fixed in other shorter-term markets or even after the time of delivery, P2P markets may require a strong technical control over transactions while they are carried out, since there no possibility to fix possible technical issues. Therefore the claim from the directive about strengthening the short-term markets becomes more realistic, although technical feasibility of blockchain solutions considering time for validation, consensus and conflicts resolution must be always assessed. Theoretically, addressing a full P2P market would imply the non-existence of the grid intended as a means to compensate for possible imbalances, leaving the full control of operations to decentralized agents. In this sense, loads and generation forecasting, as well as local availability of storage units, would enable the implementation of full P2P electricity exchanges and make full use of the blockchain technology. Finally, the new electricity market in Europe should be as much as possible 'integrated' and efficient. As far as integration is concerned, the same rules for price formation should be shared and agreed upon and this could be easily done using a transparent business integration tool supported by the same protocol (i.e.: blockchain technology). As far as efficiency is concerned, the values of all assets at any instant should reflect all available information. In an efficient market place indeed, the suppliers and buyers together adjust product price immediately in real-time to the new information available. Both features can be attained using the blockchain technology as enabler of B2B and B2C integration and transparency in data exchange, although the blockchain trilemma advises against highly secure blockchain platforms for highly scalable and distributed applications. Table 5 summarizes the principal features involved in energy market issues.

Table 5. Energy markets.

\begin{tabular}{|c|c|c|c|}
\hline & Spot markets & $\begin{array}{c}\text { Bilateral (regulated } \\
\text { and OTC) }\end{array}$ & Blockchain and P2P \\
\hline $\begin{array}{c}\text { Control over energy } \\
\text { transactions }\end{array}$ & centralized & decentralized & decentralized \\
\hline $\begin{array}{c}\text { Number of } \\
\text { participants }\end{array}$ & Many & 2 & $\begin{array}{c}\text { Those taking part in the } \\
\text { BC community }\end{array}$ \\
\hline $\begin{array}{c}\text { Conditions for } \\
\text { entering the market }\end{array}$ & Yes & Yes & No \\
\hline Participants & None & $\begin{array}{c}\text { Between the two } \\
\text { participants }\end{array}$ & $\begin{array}{c}\text { Verified by the } \\
\text { authentication mechanism }\end{array}$ \\
\hline
\end{tabular}




\begin{tabular}{|c|c|c|c|}
\hline transparency & & & \\
\hline Timing & Mostly Daily or Weekly & Mostly Hourly & $15^{\prime}$ to hourly \\
\hline Trading & $\begin{array}{l}\text { Through the Market } \\
\text { platform }\end{array}$ & $\begin{array}{l}\text { Directly or through a } \\
\text { trader }\end{array}$ & Directly \\
\hline Price setting & $\begin{array}{c}\text { Merit order or } \\
\text { Demand/Offer agreement } \\
\text { or 'pay as bid' when } \\
\text { needed }\end{array}$ & $\begin{array}{l}\text { Influenced by exchange } \\
\text { markets }\end{array}$ & $\begin{array}{c}\text { Influenced by exchange } \\
\text { markets }\end{array}$ \\
\hline Price transparency & To all & $\begin{array}{c}\text { To those who take part in } \\
\text { the transaction }\end{array}$ & $\begin{array}{l}\text { To those taking part in the } \\
\text { BC community }\end{array}$ \\
\hline Counterparty Risks & Minimum & High & Minimum \\
\hline Need for balancing & Strong & Strong & Limited $^{2}$ \\
\hline $\begin{array}{l}\text { Contracts and } \\
\text { products }\end{array}$ & $\begin{array}{c}\text { Standardized and } \\
\text { subjected to precise rules }\end{array}$ & Not standardized & Not standardized \\
\hline
\end{tabular}

\subsection{Challenges}

Although rich of potential, the blockchain technology application in the electrical power sector still poses big challenges to technicians. Innovation and experimentation are required essentially across technology and application. On the technology side, some of the limitations are well known, i.e. scalability, speed and the high energy costs associated with bitcoin and public proof of work-based blockchains. Security is another issue, since blockchain's security remains unproven until it has grown enough to be attractive to cyber attackers. Another risk concerns the lack of flexibility once blockchains are deployed as significant stakeholder buy-in is required before large upgrades can be made. In addition, there are unresolved user-friendliness and user privacy requirements regarding key management and data management. Potential application challenges concern the structure of the electricity industry and scope in network operation. These issues may jeopardize the growth of certain blockchain-based applications. P2P communities are indeed unlikely to operate independently from grid operators as long as they are connected to the central grid due to the reconciliation requirements. Another big challenge concerns the energy consumption for the execution of energy-consuming consensus algorithms, such as the proof-of-work. A recent study from the Life Cycle Assessment expert Anders S.G. Andrae [75] reports that in 2015 ICT is responsible for 1757 TWh of world energy consumption. This latter number is expected to be doubled by 2025 in the best case scenario and tripled in the worst case scenario. The study does not explicitly mention blockchain that, if entered into widespread use, could create an even more impacting situation. Finally there are alternative technological solutions for many of the potential applications being explored by projects, which blockchain may not necessarily outperform. For example, one alternative is algorithm-based, such as Open Utility's Piclo power matching platform, while telemetry-based systems are another. The public perception challenge also is highlighted, with blockchain only recently starting to gain public legitimacy and the risk of disillusionment if implementations fail to deliver.

\footnotetext{
${ }^{2}$ Under certain smart contracts conditions.
} 


\section{Conclusions}

Among all the sectors of application of the blockchain technology, the electrical energy sector seems the most promising, probably because, intrinsically, electrical energy shares some characteristics with electronic money: it is fungible, convertible in various forms, distributed through networks and infrastructures and cannot be cloned with a simple copy/paste. If the goal is to develop a transparent, secure and disintermediated exchange of goods, electricity seems to be the ideal type of asset to be managed through the blockchain technology. In this new era of digitization, the business model relating to the ownership of physical resources will not be affected much, but the network operation will instead. This paper offers a wide perspective over the main technical challenges in applying the blockchain technology to electricity trading, tracing and certification. These latter are considered as two classes of applications for which different features of blockchain technologies seem more relevant. This paper also offers a wide perspective over smart contracts implementation under different blockchain technologies in the power systems area, for which they have special features that relate to the underlying infrastructure and type of trading that cannot be neglected. Time dimension and data privacy are two items that must be handled with care when considering electricity and blockchain applications and this paper reviews them in the field of power systems applications.

Acknowledgments: The authors wish to thank Eng. Michele Fiorelli for his valuable contribution in the revision of some parts of this work related to the energy market.

Conflicts of Interest: The authors declare no conflict of interest.

\section{References}

[1] S. Nakamoto, Bitcoin : A Peer-to-Peer Electronic Cash System, (2008) 1-9.

[2] R.J. Maly, Comparison of Centralized (Client-Server) and Decentralized ( Peer-to-Peer ) Networking, Semester thesis, University of Zurich (2003). https://pub.tik.ee.ethz.ch/students/2002-2003-Wi/SA-2003-16.pdf, (accessed December 04, 2018).

[3] M. Jabed, M. Chowdhury, A. Colman, M.A. Kabir, J. Han, P. Sarda, Blockchain versus Database : A Critical Analysis, 2018 17th IEEE International Conference On Trust, Security And Privacy In Computing And Communications/ 12th IEEE International Conference On Big Data Science And Engineering, (2018).

[4] D. Gruyter, Potential of the Blockchain Technology in Energy Trading, (2020) 1-44.

[5] EPEX SPOT markets: focus on flexibility 23/01/2019, (2019).

[6] IBM Institute for Business Value, Blockchain reinvents the consumer experience, executive report. IBM Institute for Business Value, Blockchain reinvents the consumer
https://www.ibm.com/downloads/cas/REGBVG7J, (accessed December 03, 2018).

[7] G. Dutchs, N. Steinecke, Use cases of blockchain technology in energy and commodity trading, PWC. (2017) https://www.pwc.com/gx/en/industries/assets/blockchain-technology-in-energy.pdf.

[8] S.K. Lo, X. Xu, Y.K. Chiam, Q. Lu, Evaluating Suitability of Applying Blockchain, in: Proc. IEEE Int. Conf. Eng. Complex Comput. Syst. ICECCS, 2018: pp. 158-161.

[9] I. Karamitsos, M. Papadaki, N. Baker, A. Barghuthi, Design of the Blockchain Smart Contract: A Use Case for Real Estate, (2018) 177-190.

[10] O. Jogunola, A. Ikpehai, K. Anoh, B. Adebisi, M. Hammoudeh, S.Y. Son, G. Harris, State-of-the-art and prospects for peer-to-peer transaction-based energy system, Energies. 10 (2017) 1-28.

[11] C. Zhang, J. Wu, C. Long, M. Cheng, Review of Existing Peer-to-Peer Energy Trading Projects, Energy Procedia. 105 (2017) 2563-2568. http://dx.doi.org/10.1016/j.egypro.2017.03.737.

[12] T. Sousa, T. Soares, P. Pinson, F. Moret, Peer-to-peer and community-based markets : A comprehensive review. http://pierrepinson.com/docs/Sousaetal2018.pdf, (accessed November 28, 2018)

[13] T. Morstyn, N. Farrell, S.J. Darby, M.D. Mcculloch, plants, Nat. Energy. (2018) http://dx.doi.org/10.1038/s41560-017-0075-y, (accessed November 30, 2018).

[14] J. Wu, N.K. Tran, Application of Blockchain Technology in Sustainable Energy Systems : An Overview, (2018) $1-22$.

[15] N. Wang, X. Zhou, X. Lu, Z. Guan, L. Wu, X. Du, applied sciences, When Energy Trading Meets Blockchain in Electrical Power System: The State of the Art. https://www.mdpi.com/2076-3417/9/8/1561/pdf, (accesed December 01,2018)

[16] M. Andoni, V. Robu, D. Flynn, S. Abram, D. Geach, D. Jenkins, P. Mccallum, A. Peacock, Blockchain technology in the energy sector: A systematic review of challenges and opportunities, Renew. Sustain. Energy Rev. 100 (2019) 143-174. https://doi.org/10.1016/j.rser.2018.10.014. 
[17] T. Yang, Q. Guo, X. Tai, H. Sun, B. Zhang, W. Zhao and C. Lin, Applyng Blockchain Technology to Decentralized Operation in Future Energy Internet, 2017 IEEE Conference on Energy Internet and Energy System Integration (2017) 1-5.

[18] G. Liang, S.R. Weller, F. Luo, Distributed Blockchain-Based Data Protection Framework for Modern Power Systems Against Cyber Attacks, IEEE Trans. Smart Grid. 10 (2019) 3162-3173.

[19] V. Brilliantova, T.W. Thurner, Technology in Society Blockchain and the future of energy, Technol. Soc. 57 (2019) 38-45. https://doi.org/10.1016/j.techsoc.2018.11.001.

[20] A. Ahl, M. Yarime, K. Tanaka, D. Sagawa, Review of blockchain-based distributed energy: Implications for institutional development Review of blockchain-based distributed energy: Implications for institutional development, (2019).

[21] M.E. Peck, Blockchains: How they work and why they'll change the world, IEEE Spectr. 54 (2017) $26-35$.

[22] Z. Li, J. Kang, R. Yu, D. Ye, Q. Deng, Y. Zhang, Consortium blockchain for secure energy trading in industrial internet of things, IEEE Trans. Ind. Informatics. 14 (2018) 3690-3700.

[23] C. Park, T. Yong, Comparative review and discussion on P2P electricity trading ScienceDirect ScienceDirect Comparative review and discussion on P2P electricity trading, Energy Procedia. 128 (2019) 3-9. https://doi.org/10.1016/j.egypro.2017.09.003.

[24] J. Kang, R. Yu, X. Huang, S. Maharjan, Y. Zhang, E. Hossain, Enabling Localized Peer-to-Peer Electricity Trading among Plug-in Hybrid Electric Vehicles Using Consortium Blockchains, IEEE Trans. Ind. Informatics. 13 (2017) 3154-3164

[25] X. Huang, C. Xu, P. Wang, H. Liu, LNSC: A Security Model for Electric Vehicle and Charging Pile Management based on Blockchain Ecosystem, (2018).

[26] T. Lee, J. Hwang, S. Kim, P. Ferrão, J. Fournier, District Blockchain Heating and Cooling Energy Prosumer Business Model on Using System to Energy Prosumer Business Model Using Blockchain System to Ensure Transparency and Safety Assessing the feasi, Energy Procedia. 141 (2017) 194-198. https://doi.org/10.1016/j.egypro.2017.11.037.

[27] C. Pop, T. Cioara, M. Antal, I. Anghel, I. Salomie, M. Bertoncini, Blockchain based decentralized management of demand response programs in smart energy grids, Sensors (Switzerland). 18 (2018).

[28] L. Xue, Y. Teng, Z. Zhang, J. Li, K. Wang, Q. Huang, Blockchain technology for electricity market in microgrid, IEEE. (2017). http://online.liebertpub.com/doi/10.1089/jpm.2015.0146.

[29] S. Thakur, B.P. Hayes, S. Member, J.G. Breslin, S. Member, Distributed Double Auction for Peer to Peer Energy Trade using Blockchains, 2018 5th Int. Symp. Environ. Energies Appl. (2018) 1-8.

[30] M. Sabounchi, A Decentralized P2P Electricity Market Model for Microgrids, 2018 IEEE Power Energy Soc. Gen. Meet. (2018) 1-5.

[31] B.C. Neagu, I. Member, G. Grigora, O. Ivanov, I. Member, An Efficient Peer-to-Peer Based Blockchain Approach for Prosumers Energy Trading in Microgrids, (2019).

[32] M.L. Di Silvestre, L. Dusonchet, S. Favuzza, M.G. Ippolito, S. Mangione, F. Massaro, L. Mineo, E.R Sanseverino, E. Telaretti, G. Zizzo, Transparency in transactive energy at distribution level, 2017 AEIT Int Annu. Conf. Infrastructures Energy ICT Oppor. Foster. Innov. AEIT 2017. 2017-Janua (2017) 1-5.

[33] M.L. Di Silvestre, P. Gallo, M.G. Ippolito, R. Musca, E.R. Sanseverino, Q.T.T. Tran, Ancillary Services in the Energy Blockchain for Microgrids, IEEE Transactions on Industry Applications, (2019), Early Access (DOI 10.1109/TIA.2019.2909496)

[34] M.L. Di Silvestre, P. Gallo, M.G. Ippolito, E.R. Sanseverino, G. Zizzo, A technical approach to the energy blockchain in microgrids, IEEE Trans. Ind. Informatics. 14 (2018) 4792-4803.

[35] L.W. Park, S. Lee, H. Chang, A sustainable home energy prosumer-chain methodology with energy tags over the blockchain, Sustain. 10 (2018) 1-18.

[36] H.S.V.S.K. Nunna, Y. Amanbek, B. Satuyeva, S. Doolla, A Decentralized Transactive Energy Trading Framework for Prosumers in a Microgrid Cluster, 2019 IEEE PES GTD Gd. Int. Conf. Expo. Asia (GTD Asia). (2019) 824-830.

[37] C. Long, J. Wu, C. Zhang, M. Cheng, A. Al-Wakeel, Feasibility of Peer-to-Peer Energy Trading in Low Voltage $\begin{array}{llllll}\text { Electrical Distribution Networks, Energy Procedia. } 105 & \text { (2017) 2227-2232. }\end{array}$ http://dx.doi.org/10.1016/j.egypro.2017.03.632.

[38] W. Ning, W. Xu, A.M. Devices, Z. Xu, Peer-to-Peer Energy Trading among Microgrids with Multidimensional Willingness, Energies. 11(12) (2018): 3312

[39] R.K. Kodali, S. Yerroju, Blockchain Based Energy Trading, TENCON 2018 - 2018 IEEE Reg. 10 Conf. (2018) $1778-1783$.

[40] S.J. Pee, J.G. Song, Blockchain based smart energy trading platform using smart contract, 2019 Int. Conf. Artif. Intell. Inf. Commun. (2019) 322-325.

[41] A. Alam, T. Islam, Towards Blockchain-based Electricity Trading System and Cyber Resilient Microgrids, 2019 Int. Conf. Electr. Comput. Commun. Eng. (2019) 1-5.

[42] S. Chen, C. Liu, From demand response to transactive energy: state of the art, J. Mod. Power Syst. Clean Energy. 5 (2017) 10-19.

[43] D. Croce, F. Giuliano, I. Tinnirello, A. Galatioto, M. Bonomolo, M. Beccali, G. Zizzo, Overgrid : A Fully Distributed Demand Response Architecture Based on Overlay Networks, (2016).

[44] P. Prosumers, N. Liu, X. Yu, C. Wang, C. Li, L. Ma, J. Lei, An Energy Sharing Model with Price-based Demand Response for Microgrids of, (2017).

[45] Y. He, H. Li, X. Cheng, Y. Liu, C. Yang, L. Sun, A Blockchain Based Truthful Incentive Mechanism for Distributed P2P Applications, IEEE Access. 6 (2018) 27324-27335.

[46] K. Gai, Y. Wu, L. Zhu, M. Qiu, M. Shen, Privacy-preserving energy trading using consortium blockchain in smart grid, IEEE Trans. Ind. Informatics. 15 (2019) 3548-3558. 
[47] D. Arnone, M. Mammina, S. Favuzza, M.G. Ippolito, E. Riva Sanseverino, E. Telaretti, G. Zizzo, DEMAND Project: Bottom-Up Aggregation of Prosumers in Distribution Networks, Proc. 2018 AEIT Int. Annu. Conf., (2018) $1-6$.

[48] S.-J. Lee, J.-H. Nam, I.-G. Lee, Erasable blockchain: A next generation blockchain for ensuring the right to be forgotten, J. Adv. Res. Dyn. Control Syst., vol. 10, no. 14 Special Issue, (2008) 257-264.

[49] M. Florian, S. Beaucamp, S. Henningsen, B. Scheuermann, Erasing Data from Blockchain Nodes. https://arxiv.org/pdf/1904.08901, (accessed July 15, 2019).

[50] X. Xu, I. Weber, M. Staples, Design Process for Applications on Blockchain, in Architecture for Blockchain Applications, Springer, (2019) 93-111.

[51] S. Ølnes, J. Ubacht, M. Janssen, Blockchain in government: Benefits and implications of distributed ledger technology for information sharing, (2017) 355-364.

[52] G. Greenspan, Smart contract showdown: Hyperledger Fabric vs MultiChain vs Ethereum vs Corda, (2018). https://www.multichain.com/blog/2018/12/smart-contract-showdown/ (accessed December 15, 2018).

[53] Nemogrid. http://nemogrid.eu/ (accessed December 12, 2018)

[54] V. Buterin, A NEXT GENERATION SMART CONTRACT \& DECENTRALIZED APPLICATION PLATFORM, Ethereum White Pap. (2009) 1-36.

[55] G. Greenspan, MultiChain Private Blockchain - White Paper, (2013) 1-17.

[56] Tendermint Documentation, (2017). https://media.readthedocs.org/pdf/tendermint/v0.10.4/tendermint.pdf (accessed December 13, 2018).

[57] hyperledger-fabricdocs Documentation, (2019). https://media.readthedocs.org/pdf/hyperledger-

fabric/latest/hyperledger-fabric.pdf (accessed July 29, 2019).

[58] V. Buterin, V. Griffith, Casper the Friendly Finality Gadget, (2018).

[59] Hyperledger Fabric Docs - Network. https://hyperledger-fabric.readthedocs.io/en/release-

1.2/network/network.html (accessed December 5, 2018)

[60] J. Guerrero, S. Member, A.C. Chapman, Decentralized P2P Energy Trading under Network Constraints in a Low-Voltage Network, (2018).

[61] A. Perez-diaz, E. Gerding, F. Mcgroarty, Decentralised Coordination of Electric Vehicle Aggregators. https://eprints.soton.ac.uk/422623/1/manuscript.pdf, (accessed December 02, 2018).

[62] E. Munsing, J. Mather, S. Moura, Blockchains for Decentralized Optimization of Energy Resources in Microgrid Networks. https://ecal.berkeley.edu/pubs/CCTA17_Blockchain.pdf, (accessed December 05, 2018).

[63] E. Mengelkamp, J. Gärttner, K. Rock, S. Kessler, L. Orsini, Designing microgrid energy markets A case study: The Brooklyn Microgrid, (2017) 10-12.

[64] Infinity Blockchain. https://www.blockchainlabs.asia/ (accessed December 15, 2018).

[65] Wien Energie, Annual review 2018, (2017). http://jahrbuch.wienenergie.at/de/2017/zgq8uTUS/wien-energie2017-die-top-10/?page=1 (accessed December 20, 2018).

[66] Tennet, Europe's first blockchain project to stabilize the power grid launches: TenneT and sonnen expect results in 2018, (2018). https://www.tennet.eu/news/detail/europes-first-blockchain-project-to-stabilize-the-power-gridlaunches-tennet-and-sonnen-expect-res/ (accessed December 19, 2018).

[67] Solara White Paper, (2018). https://solara.io/wp-content/uploads/2018/06/Solara-WP-Public-Release-Candidatev1.4.8-6th-June-2018-LGV-Signed.pdf (accessed December 16, 2018).

[68] D. Carboni, Incognite e attrattive della blockchain nel settore energetico, (2018).

[69] M. Buchmann, Blockchain technology - a threat to distribution network operators?, (2018). https://energypost.eu/blockchain-technology-a-threat-to-distribution-network-operators (accessed December 10, 2018).

[70] Utilising the Blockchain in today's OTC energy trading, (2016).

https://www.advisoryhouse.com/images/TAHContent/Fokusartikel/TAH Zentrale Erzeugung Handel Speicher Blockchain.pdf (accessed December 18, 2018).

[71] ENERCHAIN - DECENTRALLY TRADED DECENTRAL ENERGY. https://enerchain.ponton.de/ (accessed December 18, 2018)

[72] Directive OF THE EUROPEAN PARLIAMENT AND OF THE COUNCIL on common rules for the interna market in electricity.

[73] H. Beitollahi and G. Deconinck Peer-to-Peer Networks Applied to Power Grid, (2014). http://citeseerx.ist.psu.edu/viewdoc/download?doi=10.1.1.134.7160\&rep=rep1\&type=pdf, (accessed December 07, 2018)

[74] Y. Parag, B.K. Sovacool, Electricity market design for the prosumer era, (2016) http://sro.sussex.ac.uk/id/eprint/60088/1/NENERGY-15100399 1455284484 11\%20-\%20FINAL.pdf, (accessed December 06, 2018)

[75] A.S.G. Andrae, Total Consumer Power Consumption Forecast, Nordic Digital Business Summit, (2017)

[76] ENTSO-E, Towards smarter grids: Developing TSO and DSO roles and interactions for the benefit of consumers, https://docstore.entsoe.eu/Documents/Publications/Position\%20papers\%20and\%20reports/150303 ENTSO-E Position Paper TSO-DSO interaction.pdf 
